\title{
An internal focus of attention is optimal when congruent with afferent proprioceptive task information
}

Gottwald, Vicky; Owen, Robin; Lawrence, Gavin; McNevin, Nancy

\section{Psychology of Sport and Exercise}

DOI:

10.1016/j.psychsport.2019.101634

Published: 01/03/2020

Peer reviewed version

Cyswllt i'r cyhoeddiad / Link to publication

Dyfyniad o'r fersiwn a gyhoeddwyd / Citation for published version (APA):

Gottwald, V., Owen, R., Lawrence, G., \& McNevin, N. (2020). An internal focus of attention is optimal when congruent with afferent proprioceptive task information. Psychology of Sport and Exercise, 47, [101634]. https://doi.org/10.1016/j.psychsport.2019.101634

\section{Hawliau Cyffredinol / General rights}

Copyright and moral rights for the publications made accessible in the public portal are retained by the authors and/or other copyright owners and it is a condition of accessing publications that users recognise and abide by the legal requirements associated with these rights. study or research.

- Users may download and print one copy of any publication from the public portal for the purpose of private

- You may not further distribute the material or use it for any profit-making activity or commercial gain

- You may freely distribute the URL identifying the publication in the public portal ?

Take down policy

If you believe that this document breaches copyright please contact us providing details, and we will remove access to the work immediately and investigate your claim. 


\section{Abstract}

2 Whilst benefits of an external focus are shown to govern several characteristics of skill execution,

3 specificity theory indicates that sources of afferent information most useful to performance

4 execution are typically prioritised during processing.

5 Objectives: We investigated whether an internal focus facilitates performance when pertinent

6 afferent information is proprioceptive in nature and congruent with attentional focus. We also

7 considered whether the mechanisms behind attentional focus differences are attributable to planning

8 processes or online motor control.

9 Design: Experiments 1 and 2 adopted a randomised design, whilst experiment 3 used a repeated measures approach.

11 Method: In Experiment 1 we investigated movement variability as a measure of planning and error correction under external and internal focus conditions in an aiming task. Experiment 2 removed visual information to increase pertinence of proprioceptive feedback for movement execution and Experiment 3 adopted a leg-extension task, where proprioceptive salience was enhanced using an ankle weight. We hypothesized that this would increase congruency between internal focus instructions and movement production. Results: Experiments 1 and 2 revealed reduced amplitude errors under an internal focus whilst Experiment 3 showed similar findings with the addition of lower EMG activity when adopting an internal focus. Movement variability findings were indicative of enhanced planning. Conclusions: When pertinence of proprioceptive information was amplified, benefits of an internal focus were more pronounced and performance was higher. Participants were better able to focus on movement characteristics to process proprioceptive feedback: something not afforded under an external focus. This raises doubts regarding the rigidity of the constrained action hypothesis. 

deliberate practice and subsequently help maximise skill acquisition (Ericsson, Krampe \& TescheRömer, 1993). Equally, recent literature has begun to identify contexts where certain instructions can impede performance. There is now a compelling body of evidence that demonstrates the advantages of adopting an external (i.e., centered toward movement effects) over internal (i.e., centred toward body movements) focus of attention, in governing several characteristics of skill execution such as accuracy, consistency, and economy (for a review see Wulf, 2013). One mechanism thought critical in accounting for these advantages is movement planning (e.g. Prinz, 1997; Lohse, Sherwood \& Healy, 2010). Whilst we are typically aware of the intended goal of particular movements, it is the responsibility of the executive motor system for the selection,

11 programming, and initiation of the action: often a complex pattern of specific muscle activities. In sporting actions, we will plan where a particular limb will finish up at the end of a movement

(Woodworth, 1899; Schmidt, Zelaznik, Hawkins, Frank, \& Quinn Jr, 1979). It is this planning process specifically, that is often suggested to account for attentional focus differences. Focus differences were first recognised during early experimental research requiring participants to focus on movement effects as opposed to body movements themselves (Wulf, Höb, \& Prinz, 1998). When this happened, performance was superior. As well as performance accuracy, advantages in attentional capacity have contributed to the development of the constrained action hypothesis (Wulf, McNevin \& Shea, 2001). This suggests that focusing on the effects of one's movements promotes automaticity in movement execution and prevents any undesirable interference in response programming and control (likely to occur as a result of focusing internally). Findings have also been accounted for with Prinz's (1997) action effect principle, which emphasizes a compatible relationship between movement planning and outcome. Thus, if actions are planned and controlled in relation to their effects, then focusing externally should facilitate performance by enhancing congruence between movement planning and the desired response. 
1 Similarly, Wulf and Lewthwaite's (2016) OPTIMAL (optimising performance through intrinsic motivation and attention for learning) theory of motor learning proposes enhanced goal-action coupling when using an external focus of attention. Offering further support for this notion, Wulf (2015) states that "adopting an external focus is related to the planning of movement, but has nothing to do with the processing of intrinsic feedback or bodily awareness, or lack thereof" (p.337). However, regardless of theoretical hypotheses, pertinent variables are yet to be rigorously tested, including the effect of focus of attention on movement planning and control mechanisms, especially in non-continuous tasks. A minority of researchers have begun to explore planning processes as a function of focus of attention, but as a secondary aim and often with methodological limitations in the approach adopted (e.g. Lohse et al., 2010; Lohse, 2012).

Work by Lohse et al. (2010) hypothesised higher levels of explicit processing when employing an internal focus of attention in a dart-throwing task. Dependent variables included a proxy measure of planning (preparation time between throws) with explicit processing expected to be reflected via increases in inter-trial intervals when adopting an internal focus. Findings were consistent with this notion and viewed as a manifestation of enhanced planning when using an external focus of attention. However, Lohse (2012) concedes the rudimentary nature of this approach to investigate planning. This measure was refined accordingly to calculate pre-movement times, this time in an isometric force production task. Pre-movement times were measured as the time from the 'go' signal to a change in recorded force of $+/-1 \mathrm{lb}$. Findings revealed lower premovement times for those adopting an external focus of attention early on in the learning process. However, attentional focus researchers have negated to consider important modifications that may be made during an action to achieve an intended goal i.e., motor control. Seminal attentional focus studies investigating balance board performance (e.g. Wulf et al., 1998; Wulf et al., 2001) imply that benefits of an external focus of attention may be due to corrections made during the movement. One could consider this to be online control and therefore less related to 
1 planning. In an aiming movement, these control mechanisms involve any movement adjustments necessary to correct discrepancies between the end of a limb's initial impulse and its target (Woodworth, 1899; Elliott, Helsen \& Chua, 2001). With both planning and control mechanisms heavily integrated, it would be remiss not to consider them in the context of one another, especially being that aiming movements incorporate both pre-planned offline (feedforward) and online (somatosensory) adjustments (Elliott, Chua, Pollock, \& Lyons, 1995; 20 \& Kawato, 1998; Khan, Elliott, Coull, Chua, \& Lyons, 2002; Elliott et al., 2014). Whilst the aforementioned findings (Lohse et al., 2010; Lohse, 2012) are in line with attentional focus hypotheses surrounding movement planning, we would argue that in the absence of motor control measures, it is difficult to fully understand information processing or 'trade-offs' between planning and control in the context of focus of attention. Ironically, findings are accounted for using Willingham's (1998) control-based learning theory (COBALT), which only reinforces the importance of investigating differences in output control as well as planning. COBALT is a neuropsychological theory of skill learning. Its framework provides support for a series of perceptual-motor processes, which occur largely outside of conscious control (i.e., goal selection), motor sequencing (i.e., planning), and muscle activation. However, target selection and planning processes can also occur as a more conscious process whereby they are coded in egocentric as opposed to allocentric space. Lohse (2012) suggests that adopting an internal focus of attention is more likely to induce this egocentric coding and thus, conscious control over actions. This is arguably more attention demanding and accounts for increased planning demands, which then manifest through longer inter-trial intervals and pre-movement times. However, Willingham (1998) proposes that both conscious and unconscious modes of skill execution can be helpful to performance.

In the absence of any investigations into movement 'control' in conjunction with planning processes, the current set of studies are the first to include rigorous measures of these mechanisms. 
1 In turn, this also provides a novel application of Khan et al.'s (2006) variability methodology, which has been shown to provide a robust measure of both planning (i.e., offline cognitive processing of feedback between trials for movement planning) and control (i.e., online adjustments made during a movement) processes. This approach has been most often adopted in rapid aiming tasks, which afford a rigorous analysis of kinematic profiles of movements. The methodological technique initially stems from Woodworth's (1899) aforementioned two-component model of goaldirected aiming (for a more recent review see Elliott et al., 2001). This provides a detailed analysis of two proposed phases of target-aiming movements: an initial impulse phase, which consists of central planning to move the limb into the vicinity of the target followed by an adjustment phase, which controls the movement using sensory feedback in order to reach the target. Measures of

variability provide useful indications of these components' relative contributions to movement execution. Whilst errors early on in an action tend to be indicative of poor motor planning, control processes typically occur online and can be reflected in error corrections made later in a movement trajectory (see Khan et al., 2006 for a review).

Furthermore, literature has also observed instances where attentional shifts towards and not away from movements have been advocated, such as process goals (Zimmerman \& Kitsantas, 1997) or external/kinaesthetic motor imagery (Hardy \& Callow, 1999). Similarly, the philosophical concept of a functional 'somaesthetic awareness' (Shusterman, 2011) has been proposed by Toner and Moran (2015) for use in making movement adjustments for error correction or when re-learning movements. This is in line with arguments made by Collins, Carson and Toner (2015) highlighting reported benefits from athletes sometimes adopting a focus towards particular aspects of a movement. They suggest that optimal focus is dependent on factors such as familiarisation with instructions, focus relevance and availability of physical implements during execution (see also Lawrence, Gottwald, Hardy, \& Khan, 2011). These movement related techniques are clearly contradictory to Wulf's persuasive recommendations cautioning against a 'movement centred' 
1 focus (see Wulf, 2013 for a review). We hypothesize that these discrepancies are likely a result of task nuances and that adopting a movement centred focus may actually add value in particular contexts yet to be explored. When performing an aiming movement, available sensory information in the form of either visual or proprioceptive and kinaesthetic information is utilised in guiding the limb to the target via offline and online motor control (Crossman \& Goodeve, 1983). According to specificity of practice hypothesis (Proteau, 1992; Coull, Tremblay, \& Elliott, 2001), the source of afferent information most useful to performance execution is typically prioritised for processing. In an aiming task where proprioception is an arguably important source of information, we might expect this to manifest via enhanced processing (e.g. planning or control) when using an internal and not external focus of attention. This is in line with the aforementioned phenomenon of a facilitative somaesthetic awareness (Shusterman, 2011). Interestingly, Porter, Wu, and Partridge (2010) reveal that $84.6 \%$ of athletes surveyed report that instructions provided in their training still induce an internal focus of attention, regardless of Wulf and colleagues' recommendations. Whilst this may be a function of undesirable coaching methods, it may also be an indication of potential benefits of an internal focus in some contexts e.g. when importance of proprioceptive information is high for task success. The current series of experiments are aimed at better understanding this research lacuna in the context of offline motor planning and online motor control mechanisms. We conducted three experiments that differed systematically in the need for proprioceptive processes to meet task goals, whilst incorporating rigorous measures of planning and control.

\section{Experiment 1}

In Experiment 1 we compared the utilisation of afferent information for offline planning and online motor control processes under different attentional focus conditions. Participants completed a computer-based rapid-aiming task, under either internal or external attentional foci. We hypothesized benefits in offline planning when adopting an internal focus of attention, in line with 
1 specificity theory. In a task of this nature, we expected greater congruence between internal compared with external focus instructions and the required movement production.

\section{Method}

\section{Participants.}

Forty participants, who reported right-hand-dominance and normal / corrected-to-normal vision, were randomised into two groups: internal focus of attention $(n=20)$ and external focus of attention $(n=20)$. G*Power (G*Power 3; Faul, Erdfelder, Lang, \& Buchner, 2007) sample size estimation deemed 14 participants per group necessary to provide power $=.8$ for the interaction between focus of attention and within movement trajectory variability when alpha $=.05$ and $\eta_{\mathrm{p}}{ }^{2}=$ .05. Thus, final sample size exceeded minimum power requirements. The experiment was conducted in accordance with institutional ethical guidelines.

\section{Apparatus and task.}

The experimental task comprised a target-directed computer-based aiming movement, performed using a handheld stylus on a Calcomp III digitising tablet $(122 \mathrm{~cm} \mathrm{x} 91.5 \mathrm{~cm} \&$ sample rate $200 \mathrm{~Hz}$ ) placed horizontally in front of participants (see figure 1). An opaque shield obscured participants' vision of their hand/stylus. Stylus position was denoted via a white circular cursor $(1 \mathrm{~cm}$ diameter) on a black background using a 37" Mitsubishi Diamond Pro monitor (refresh rate $=$ $85 \mathrm{hz}$ ) located $33 \mathrm{~cm}$ in front of the participant and $20 \mathrm{~cm}$ above the digitizing tablet. The $\mathrm{X} / \mathrm{Y}$ movement of the stylus on the tablet plane corresponded 1-to-1 to the X/Y movement of the cursor on the monitor plane. A green circular 'start' marker (1cm diameter) was located at the bottom of the monitor. Three circular 'target' markers (red, $1 \mathrm{~cm}$ diameter) were each displayed $20 \mathrm{~cm}$ centreto-centre from the start position marker; one target was directly above the start marker and the other two targets were at $10^{\circ}$ to either side (angle subtended from the home position). The rationale for including multiple targets was purely to increase task complexity and thus for analysis purposes, data was later collapsed across targets. To begin each trial, participants steadied their cursor over 
1 the start marker, after which one of the targets turned green. Following a random 500-1500ms delay, a GO-tone signalled participants to initiate movement towards the target. Participants were instructed to: (1) make their movements as smoothly as possible; (2) stay within a criterion movement time (MT) of 400-500ms to allow sufficient time for processing of visual information (Carlton, 1992; Khan et al., 2003) ${ }^{1}$; and (3) be as accurate as possible in stopping their cursor over the target - therefore giving the task a directional and amplitude component. Once participants had come to a complete stop, the target became red again and participants had to return to the start position, ready for the next trial; concurrently, knowledge of results in the form of MT and a point score (see Allsop, Lawrence, Gray \& Khan, 2017) was presented on the monitor ${ }^{2}$. It was explained to participants at the beginning of testing that they were not required to make movements as fast as possible and that as long as they moved within $400-500 \mathrm{~ms}$, their performance would be determined repeated until correct. hand, and participants in the external group were instructed to focus on the fluid motion of the pen. Participants were given attentional focus reminders every 10 trials. Participants completed three

\footnotetext{
${ }^{1}$ Based on the rationale for including a MT criterion, we have not included any MT analysis within dependent measures and results sections.

2 The point score was a direct measure of performance and calculated using a combination of how close the participant was to meeting the criterion MT (in terms of absolute error) and how close their movement finished in relation to the target (end-point error). A maximum of five points were possible for each of the two components, meaning a maximum of 10 points were possible on any one trial. The maximum score of 10 was achieved if MT fell within \pm 10 ms of the criterion MT and cursor error fell within $\pm 5 \mathrm{~mm}$ of the criterion target distance. These points reduced by one whole integer for every additional $\pm 10 \mathrm{~ms}$ and every additional $\pm 5 \mathrm{~mm}$ that the cursor fell outside of the criterion MT and the criterion target distance, respectively.
} 
experimental phases: acquisition (90 trials), retention (30 trials), and transfer (30 trials). Retention trials required participants to perform the same task with no focus reminders. The transfer task was the same as retention, but targets were located $25 \mathrm{~cm}$ as opposed to $20 \mathrm{~cm}$ from the start position. Target order was randomised within each block of trials, under the condition that the same target could not appear twice in a row.

\section{Data reduction and dependent measures.}

Displacement data was filtered using a second-order dual-pass Butterworth filter with a $10 \mathrm{~Hz}$ low-pass cut-off frequency. A two-point central finite difference algorithm was used to calculate instantaneous velocity data from displacement data. This process was repeated to calculate acceleration data from velocity data. Movement initiation was defined as the point at which the cursor moved $2 \mathrm{~mm}$ from the start position. Movement end was defined as the point where absolute velocity of the stylus fell below $1 \mathrm{~mm} / \mathrm{s}$ : thus prohibiting reversal movements. The $100 \%$ start-totarget distance was defined as the point at which the cursor crossed the invisible arc subtended by the three targets. Amplitude error comprised the stylus' orthogonal deviation ( $\mathrm{mm})$ from the same invisible arc. Overshoots were numerically positive whilst undershoots were numerically negative. Directional error comprised the stylus' orthogonal deviation ( $\mathrm{mm}$ ) from the longitudinal axis connecting the home and target marker. Deviation to the left of this axis was numerically negative whilst deviation to the right of this axis was numerically positive. Trials whose amplitude or directional error deviated by $>2$ SD from the given participant's trial block mean for $>25 \%$ of the movement distance were removed prior to analysis (4.40\% of all trials were removed).

Outcome performance was inferred from constant error (CE), absolute error (AE), and variable error (VE): each calculated once from amplitude error (at movement end) and once from directional error (at 100\% start-to-target distance). CE and VE respectively comprised the withinparticipant mean and standard deviation of error. AE comprised the within-participant mean of absolute error. Planning and online motor control's role in the production of outcome performance 
1 was inferred from variability (i.e., within-participant standard deviation) throughout amplitude and directional components of movement. Amplitude variability was calculated at peak acceleration

3 (pka), peak velocity (pkv), peak negative acceleration (pkna), and movement end (end) from the

4 cursor's numerical orthogonal deviation in amplitude $(\mathrm{mm})$ from the start position. Directional

5 variability was calculated from directional error, at 25, 50, 75, and $100 \%$ of the start-to-target distance. Offline planning efficacy was inferred from variability at pka to pkv in the amplitude component, and from variability at $25 \%$ of the start-to-target distance in the directional component.

8 Corrective feedback loops should have had insufficient time to initiate before these points and 9 therefore improved planning efficacy can be inferred from reduced variability (Khan et al., 2006). Online motor control efficacy was inferred from changes in variability between pkv, pkna, and end for the amplitude component and changes in variability between $75 \%$ and $100 \%$ of movement distance for the directional component. In line with variability methodology, this 'deceleration' typically reflects corrective processes via the 'braking of the limb in order to accurately home in on the target'. Improved online motor control efficacy can be inferred from reductions in variability between these points (Khan et al., 2006). Finally, to rule out speed-accuracy trade-offs, MT (ms) (time interval between movement start and movement end) was calculated and compared between focus conditions. purposes only (as opposed to any theoretical hypotheses), data were collapsed across targets.

21 Outcome performance was inferred from separate 2 Focus of Attention (external and internal) x 3 Trial Block (acquisition, retention, and transfer) ANOVAs on endpoint amplitude and directional CE, AE, and VE (i.e., 6 omnibus ANOVAs). Offline planning's contribution to outcome performance in the amplitude component of movement was inferred from a 2 Focus of Attention 
pkv) ANOVA on amplitude component VE. Offline planning's contribution to outcome performance in the directional component of movement was inferred from a 2 Focus of Attention (external and internal) x 3 Trial Block (acquisition, retention, and transfer) ANOVA on directional component VE at 25\% movement distance. Online control's contribution to outcome performance in the amplitude component of movement was inferred from a 2 Focus of Attention (external and internal) x 3 Trial Block (acquisition, retention, and transfer) x 3 Marker (pkv, pkna, and end) ANOVA on amplitude component VE. Online control's contribution to outcome performance in the directional component of movement was inferred from a 2 Focus of Attention (external and internal) x 3 Trial Block (acquisition, retention, and transfer) x 2 Distance (75 and 100\%) ANOVA on directional component VE. Greenhouse-Geisser corrected tests are reported when the sphericity assumption was violated in omnibus analyses. Significant omnibus interactions were broken down using planned repeated contrasts.

\section{Results}

\section{Outcome performance}

Amplitude component. The results of the 3 Trial Block x 2 Focus of Attention ANOVAs on endpoint amplitude $\mathrm{CE}, \mathrm{AE}$, and $\mathrm{VE}$ are shown in Table 1B. Analysis of CE revealed a significant main effect for focus of attention and a significant Trial Block x Focus of Attention interaction. The significant focus of attention main effect revealed that the internal group generally had less endpoint amplitude CE than the external group. Breakdown of the Trial Block x Focus of Attention interaction revealed the internal group significantly decreased whilst the external group significantly increased their CE from acquisition to immediate retention $\left(F_{1,38}=4.34, p=.044, \eta_{\mathrm{p}}^{2}\right.$ $=.10)$ but not significantly from immediate retention to transfer $\left(F_{1,38}=2.20, p=.146, \eta_{\mathrm{p}}^{2}=.06\right)$ (see Figure 2). Analysis of AE revealed a significant main effect for focus of attention and a significant Trial Block x Focus of Attention interaction. The focus of attention main effect revealed that the internal group generally had less endpoint amplitude AE than the external group. 
1 Breakdown of the Trial Block x Focus of Attention interaction revealed a significant interaction from acquisition to immediate retention and a non-significant interaction from immediate retention to transfer $\left(F_{1,38}=4.10, p=.050, \eta_{\mathrm{p}}^{2}=.10 ; F_{1,38}=2.05, p=.161, \eta_{\mathrm{p}}{ }^{2}=.05\right.$, respectively $)$. Analysis of VE revealed only the main effect for group as significant: wherein the internal group generally had less endpoint amplitude VE than the external group.

Directional component. The results of the 3 Trial Block x 2 Focus of Attention ANOVAs on $100 \%$ start-to-target distance CE, AE, and VE are shown in Table 1B. Analysis of CE revealed no significant main effects nor interaction. Analysis of AE revealed only the main effect for trial block as significant. Breakdown of this main effect revealed no significant change from acquisition to immediate retention but a significant increase from immediate retention to transfer $\left(F_{1,38}=.15, p\right.$ $=.699, \eta_{\mathrm{p}}^{2}<.01 ; F_{1,38}=18.46, p<.001, \eta_{\mathrm{p}}^{2}=.33$, respectively). Analysis of $\mathrm{VE}$ also revealed only the main effect for trial block as significant. Breakdown of this main effect revealed no significant change from acquisition to immediate retention but a significant increase from immediate retention to transfer $\left(F_{1,38}=.79, p=.379, \eta_{\mathrm{p}}^{2}=.02 ; F_{1,38}=13.85, p=.001, \eta_{\mathrm{p}}{ }^{2}=.27\right.$, respectively $)$.

Insert Figure 2 here

\section{Offline planning / online control contributions to outcome performance.}

Amplitude component. The results of the 2 Focus of Attention x 3 Trial Block x 2 Marker ANOVA on amplitude variability to infer offline planning efficacy are shown in Table 1d. This analysis revealed a significant main effect for focus of attention and no significant interaction involving focus of attention. The results of the 2 Focus of Attention x 3 Trial Block x 3 Marker ANOVA on amplitude variability to infer online motor control efficacy are also shown in Table 1D. This analysis revealed a significant main effect for focus of attention and no significant interaction involving focus of attention. Overall, the presence of a significant main effect for focus of attention in both analyses (wherein an internal focus produced less variability than an external focus) and 
1 absence of significant interactions involving focus of attention, suggest that changes in offline planning and not online control were the primary contributor to the internal focus group's superior endpoint amplitude performance.

Directional component. The results of the 2 Focus of Attention x 3 Trial Block ANOVA on directional variability to infer offline planning efficacy are shown in Table $1 \mathrm{~d}$. This analysis revealed no significant focus of attention main effect and no significant interaction involving focus of attention. The results of the 2 Focus of Attention x 3 Trial Block x 2 Distance ANOVA on directional variability to infer online control efficacy are shown in Table 1D. This analysis also revealed no significant focus of attention main effect and no significant interaction involving focus of attention. In sum, no significant offline planning and online control differences emerged between focus of attention groups in the directional component of movement.

Insert Table 1 here

\section{Discussion}

Experiment 1 employed a novel use of variability methodology to investigate planning and control mechanisms under an internal and external focus of attention. This was carried out in a task where proprioceptive salience was hypothesized to be high. Participants performed a target-aiming task, during which key kinematic variables indicative of motor planning and control processes could be measured (see Khan et al., 2006). We hypothesized that the proprioceptive nature of the task may contribute to atypical findings based on the philosophical concept of a functional 'somaesthetic awareness' (Shusterman, 2011): wherein the increased congruence between task-relevant information and an internal focus of attention would yield performance and processing benefits compared to an external focus.

Findings confirmed that participants adopting an internal focus were more accurate in their movements comparative to those adopting an external focus. This was manifested via reduced 
errors throughout all experimental phases as well as decreasing levels of error between acquisition and retention. Participants adopting an external focus displayed the opposite pattern of results. Furthermore, variability findings were reflective of more consistent (i.e., less variable) movements under an internal focus of attention. It is likely that in a rapid-aiming target-directed movement, higher levels of variability may be detrimental to movement accuracy. This is inconsistent with Lohse et al. (2010) where enhanced performance was manifested by increased variability. However, Bernstein (1967) suggests that movements are constrained to the point that they are most functional. The presence of a focus of attention main effect for amplitude variability, but absence of significant interactions involving focus of attention (which would be indicative of movement adjustments made during the movement), suggests that the primary contributor to improved endpoint amplitude performance of the internal group was offline planning.

Thus, an internal focus of attention may possess some facilitative attributes when performing a task of this nature, where fine motor adjustments are required (whether made during a movement or prior to). The results of Experiment 1 are likely a reflection of unequal levels of proprioceptive information available under different focus conditions in a task of this manner. Participants adopting an internal focus of attention may have been better afforded proprioceptive information (i.e., somaesthetic awareness) due to the nature of their instructions. This important role of attentional focus in skill refinement has been deliberated previously in a series of commentaries (see Toner \& Moran, 2015, Wulf, 2015; Toner \& Moran, 2016), which propose that focusing on the body can be of benefit for refining skills and making movement adjustments. It is therefore plausible that an internal focus may possess the required characteristics for these error-correction mechanisms to occur most effectively. This benefit may be further enhanced within tasks that are more 'proprioceptive' in nature.

However, it might be argued that a rapid aiming task with both visual and proprioceptive information available was not truly proprioceptive in nature and makes it difficult to determine the 
extent to which discrete modes of information (i.e., visual versus proprioceptive awareness), were most useful to participants' motor execution when adopting different attentional foci. Thus, the rationale behind Experiment 2 was to remove this confound by applying task constraints which accentuated the significance of proprioceptive information for successful performance. This was to confirm whether tasks where proprioception is integral to performance, benefit from adopting an internal compared to external focus of attention.

\section{Experiment 2}

In aiming tasks, available sensory information through vision or proprioception is fundamental for successful movement execution (Crossman \& Goodeve, 1983). As previously mentioned, specificity of practice hypothesis (Proteau, 1992; Coull et al., 2001) proposes that the source of afferent information deemed most useful to successful performance is usually prioritised for processing. Identifying the source of this information is helpful in informing both theoretical advancement as well as the development of practical performance and learning interventions (Toussaint, Meugnot, Badets, Chesnet, \& Proteau, 2016). Experiment 2 aimed to remove the confound of visual information from Experiment 1 and provide robust evidence for the benefits of an internal focus when congruent with sources of task relevant afferent information available. Otherwise, the task remained unchanged.

We hypothesised that if those adopting an internal focus are indeed able to process proprioceptive information more readily and in line with specificity theory (Proteau, 1992), then we would expect to see a further benefit of an internal focus under no vision conditions. Khan et al. (2006) suggest that variability profiles differ depending on afferent information available. It is thus plausible that the availability of visual feedback will modulate focus of attention effects on movement variability.

\section{Methods}


The only difference between Experiment 2 and Experiment 1's methodological approach

was that vision of the cursor on the screen was removed at the beginning of each trial until movement end (e.g. consistent with Chua \& Elliott, 1993; Khan et al., 2003). Targets remained visible throughout. Participants were again randomised into two groups; internal $(n=20)$ and external $(n=20)$ and provided with the same instructions as per Experiment 1. Sample size was based on the estimation detailed in Experiment 1. Of all trials, $3.03 \%$ were identified as outliers and removed prior to analysis. Again, target data was collapsed based on experimental hypotheses.

\section{Results}

\section{Outcome performance.}

Amplitude component. The results of the 3 Trial Block x 2 Focus of Attention ANOVAs on endpoint amplitude CE, AE, and VE are shown in Table 2B. Analysis of CE revealed a significant main effect for trial block and focus of attention. Breakdown of the main effect for trial block revealed CE generally increased (i.e., greater overshoot) from acquisition to immediate retention $\left(F_{1,38}=4.94, p=.032, \eta_{\mathrm{p}}{ }^{2}=.12\right)$ and decreased from immediate retention to transfer $\left(F_{1,38}=42.54\right.$, $\left.p<.001, \eta_{\mathrm{p}}^{2}=.53\right)$. To break down the main effect for focus of attention, one sample t-tests comparing each group's mean $\mathrm{CE}$ across trial blocks to 0 were performed. This analysis revealed the external group generally undershot the target $(t(19)=-1.87, p=.078,95 \%$ CI $[-32.20,1.85])$ and the internal group generally overshot the target $(t(19)=4.11, p=.001,95 \%$ CI $10.44,32.13])$. Analysis of AE revealed only the Trial Block x Focus of Attention interaction as significant. Breakdown of this interaction revealed that there was no interaction between acquisition and immediate retention $\left(F_{1,38}=2.08, p=.157, \eta_{\mathrm{p}}^{2}=.05\right)$, but between immediate retention and transfer, the external group significantly increased whilst the internal group decreased their $\mathrm{AE}\left(F_{1}\right.$, $\left.38=11.61, p=.002, \eta_{\mathrm{p}}^{2}=.23\right)$. Analysis of VE revealed only the main effect for trial block as significant. Breakdown of this trial block revealed that generally VE increased significantly from 
1 acquisition to immediate retention $\left(F_{1,38}=17.64, p<.001, \eta_{\mathrm{p}}^{2}=.32\right)$ and from immediate retention to transfer $\left(F_{1,38}=9.38, p=.004, \eta_{\mathrm{p}}^{2}=.20\right)$.

Directional component. The results of the 3 Trial Block x 2 Focus of Attention ANOVAs on $100 \%$ start-to-target distance CE, AE, and VE are shown in Table 2B. Analysis of CE revealed no significant main effects nor interaction. Analysis of AE revealed a significant main effect for trial block and focus of attention. The focus of attention main effect revealed that generally, the internal group had less AE than the external group (see Figure 3). Breakdown of the main effect for trial block revealed that generally $\mathrm{AE}$ significantly increased from acquisition to immediate retention $\left(F_{1,38}=8.32, p=.006, \eta_{\mathrm{p}}^{2}=.18\right)$ and from immediate retention to transfer $\left(F_{1,38}=7.54, p\right.$ $\left.=.009, \eta_{\mathrm{p}}^{2}=.17\right)$. Analysis of $\mathrm{VE}$ revealed a significant main effect for trial block and a nearsignificant main effect for focus of attention. The main effect for focus of attention trended towards the internal group exhibiting less VE than the external group. Breakdown of the main effect for trial block revealed that generally AE significantly increased from acquisition to immediate retention $\left(F_{1,38}=17.64, p<.001, \eta_{\mathrm{p}}{ }^{2}=.32\right)$ and from immediate retention to transfer $\left(F_{1,38}=9.38, p=.004\right.$, $\left.\eta_{\mathrm{p}}^{2}=.20\right)$

\section{Insert Figure 3 here}

\section{Offline planning / online control contributions to outcome performance.}

Amplitude component. The results of the 2 Focus of Attention x 3 Trial Block x 2 Marker ANOVA on amplitude variability to infer offline planning efficacy are shown in Table 2D. This analysis revealed no significant main effects nor interactions involving focus of attention. The results of the 2 Focus of Attention x 3 Trial Block x 3 Marker ANOVA on amplitude variability to infer online motor control efficacy are also shown in Table 2. This analysis also revealed no significant main effects nor interactions involving focus of attention. In sum, within the amplitude 
component we observed no differences in offline planning / online control based on focus of attention.

Directional component. The results of the 2 Focus of Attention x 3 Trial Block ANOVA on directional variability to infer offline planning efficacy are shown in Table 2D. This analysis revealed a significant focus of attention main effect and no significant interaction involving focus of attention. The results of the 2 Focus of Attention x 3 Trial Block x 2 Distance ANOVA on directional variability to infer online control efficacy are shown in Table 2 . This analysis revealed a significant focus of attention main effect and a significant Focus of Attention x Trial Block x Distance interaction. The focus of attention main effect revealed that the internal focus group generally had less VE than the external focus group. Breakdown of the Focus of Attention x Trial Block x Distance interaction revealed that from immediate retention to transfer only, the external group increased their VE less between 75 and $100 \%$ movement distance than the internal group $\left(F_{1}\right.$, $\left.38=4.69, p=.037, \eta_{\mathrm{p}}^{2}=.11\right)$ : at first glance suggesting increased online correction in the external group at transfer. However, given (1) this effect being exclusive to transfer, (2) transfer likely increasing task difficulty compared to immediate retention, and (3) the internal group exhibiting superior planning and outcome performance compared to the external group, the external focus group likely exhibits increased online control at transfer because worse planning provides greater scope for correction: rather than greater corrections being a specific benefit of an external focus of attention. In sum, the consistent focus of attention main effects and absence of Focus of attention $\mathrm{x}$ Distance interaction across trial blocks, suggest that offline planning likely accounts for focus of attention differences in outcome performance.

\section{Discussion}


The rationale behind Experiment 2 was to investigate motor programming strategies under conditions of enhanced afferent information in the mode of proprioception. This was the first experiment to consider both planning and control mechanisms as a function of focus of attention in a truly proprioceptive task. In line with Experiment 1 findings, we hypothesised enhanced accuracy for those adopting an internal as opposed to external focus. This was as a consequence of enhanced congruence between an internal focus of attention and afferent information available in the form of proprioception. Additionally, it was expected that increased movement accuracy would be coupled with evidence of enhanced planning strategies under an internal focus. To test this, Experiment 2 applied task constraints through the removal of visual information to accentuate the significance of proprioceptive information for successful performance.

Error data provided further support for Experiment 1 findings, with some nuances. Amplitude CE data suggests that participants tended to make greater errors and overshoot the target when using an internal focus, whilst those adopting an external focus tended to undershoot with smaller errors. However, amplitude AE data provides a clearer picture during later experimental phases, with the external group increasing error between retention and transfer and the internal group showing the converse pattern of results. Amplitude VE findings reinforce this pattern of results, showing a marginally significant main effect $(p=.05)$ for focus of attention, with those adopting an internal focus displaying more consistent movements. Furthermore, directional variability findings suggest that the primary contributor to the improved directional outcome performance of the internal group was increased efficacy of offline planning. This was reflected in decreased directional variability early on in movement trajectories and supports the notion of a facilitative somaesthetic awareness. It should however be noted that benefits of end-point amplitude accuracy were accounted for only in directional and not amplitude variability findings. It might be that an absence of amplitude variability findings were a consequence of differences in the 'functionality' of variability between an internal and external focus. This may account for no 
1 differences here in line with Berstein (1967) and Lohse et al. (2010) degrees of freedom.

2 Experiments 1 and 2 differed in the extent to which the task emphasised the importance of

3 proprioceptive information for performance success. This was achieved through the availability of

4 vision. However, experiment 3 allowed us to manipulate the strength or 'potency' of proprioceptive

5 feedback. We felt that this would provide more robust evidence for an argument recommending that 6 focus of attention should be congruent with afferent information pertinent for task success.

\section{Experiment 3}

Experiment 2 lends support to the notion that an internal attentional focus may be effective for both movement planning and accuracy when performance-relevant afferent information primarily stems from proprioception. Experiment 3 sought to provide further evidence for this by manipulating proprioceptive salience. Participants had to encode body (i.e., leg) positions and reproduce them as accurately as possible.

This task was adopted from Toussaint et al.'s (2016) methodology, which investigated legpositioning recall, where the availability of proprioceptive information was modified in an effort to prevent neglect of proprioceptive feedback. In this case, proprioceptive information was manipulated by attaching small weights to the ankle of participants. Findings revealed that when both vision and proprioception were available, proprioceptive information was neglected and vision was the dominant force in producing accurate movements. However, the addition of ankle weight increased 'proprioceptive strength' during the task, thus reducing proprioceptive neglect. The current experiment aimed to investigate whether different attentional foci facilitate the utilisation of proprioceptive information to reproduce accurate movements and whether modifying the strength of proprioceptive information would encourage greater congruence between participants' internal focus of attention and the goal of the task.

Electromyography (EMG) of the quadriceps was also measured to provide a rigorous measure of movement efficiency. Afferent information was manipulated in different ways. Firstly 
1 vision was removed. Toussaint et al.'s (2016) work emphasizes the dominant role of visual feedback in goal directed movements when available and thus, removal of this allowed researchers to isolate proprioceptive information. Secondly, it was felt that the nature of this task better allowed for participants to code the target location proprioceptively compared to Experiments 1 and 2: further increasing proprioception's relevance to movement accuracy. Thirdly, based on the findings of Toussaint and colleagues, weight was added to the ankle of one group in an effort to increase the strength of proprioceptive information available during the task.

Thus, the rationale for the final experiment was to investigate whether manipulating the congruence between an internal focus of attention and the afferent information available, enhances the extent to which movements are executed successfully. It was hypothesised that, in line with Experiments 1 and 2, an internal focus would facilitate performance by directing participants' attention to task-relevant proprioceptive information. Furthermore, it was expected that attentional focus differences would be more pronounced in the group performing with an ankle weight due to increased potency of proprioceptive information.

\section{Methods}

\section{Participants.}

The makeup of participants in Experiment $3(n=40)$ replicated that of Experiments 1 and 2, other than an additional participation criterion being no history of nervous or muscular disorders. Participants were randomised into weight $(n=20)$ and no-weight groups $(n=20)$. Sample size was again based on the estimation detailed in Experiment 1.

\section{Apparatus.}

Participants were seated in the middle of a 12-camera volume (Vicon Nexus, sampling at $240 \mathrm{hz}$ ) on a chair modified to minimise camera obstruction and allow participants' non-dominant leg to move freely and comfortably around the knee joint. As in Toussaint et al. (2016) and Toussaint and Blandin (2010), participants' non-dominant leg was used to perform the task: to 
reduce the chance of a ceiling effect and increase task novelty (Beilock, Carr, MacMahon, \& Stalkes, 2002). The weight group had a $0.5 \mathrm{~kg}$ sand-bag wrapped tightly around the base of their non-dominant leg's ankle, whilst the no-weight group did not. Leg movement in 3D space was captured via the coordinates of four reflective markers: one placed on the lateral condyle, medial condyle, lateral malleolus, and medial malleolus. A custom Microsoft Visual Basic script computed a live mid-point between the condyle markers, and between the malleolus markers, respectively. From these midpoints, a digital plane was computed to represent the lower leg position. At the beginning of testing, participants were asked to let their leg hang freely and relaxed. This position denoted their start position in every trial and a wooden box was placed so their heel gently touched the front face of the box when in this position (to aid start-position consistency). Before every trial, the experimenter ensured participants' leg was within $+/-1$ degree of the starting position. Leg angle was calculated live (at 240hz) as the difference in position between the start position and the plain representing their lower leg. Single-differential electromyography (EMG) surface electrodes (DE 2.1, Delsys, Boston, MA) were placed on the vastus medialis and vastus lateralis of participants' non-dominant leg to provide an electromyographical measure of leg extension efficiency (Alkner, Tesch, \& Berg, 2000). Electrode placement and site preparation was in line with SENIAM recommendations (Hermens et al., 1999). Each EMG electrode's signal was 10k amplified (Bagnoli-2, Delsys) and digitized at $2500 \mathrm{~Hz}$ using a computer running Spike2 software (Cambridge Electronic Design).

\section{Experimental procedure and design.}

Every trial comprised an encoding and recall phase respectively. For the encoding phase, participants were instructed to slowly extend their leg from the start position until a tone sounded indicating participants were within $+/-1^{\circ}$ of the target position. Participants then had to hold and mentally encode this angle for 2 seconds. Once the tone had sounded for 2 seconds: the precise leg position was recorded as the 'encoded position'; the tone changed to a higher pitch; and participants 
1 had to return to the start position. Upon participants' return to the start position, following a random foreperiod between 1.5 and 2.5 seconds, the recall phase was initiated via a GO-tone for movement initiation. For the recall phase, participants were instructed to: reproduce the target angle as accurately as possible (basing task exclusively on the amplitude); produce one smooth and straight movement within a MT between 500 and $1000 \mathrm{~ms}$; not concern themselves with their reaction time; and hold their recalled position for 1 second at the end, before returning to the start position (ready for the next trial) (see figure 4). Following each trial, participants were provided with knowledge of results on a monitor in front of them; this consisted of recall phase error (i.e., by how many degrees

they had undershot or overshot the target position) and MT (i.e. the ms time interval between movement initiation and end). If trials had a MT $<500 \mathrm{~ms}$ or $>1000 \mathrm{~ms}$, they were repeated until correct. As in Toussaint et al. (2016), participants were not permitted to open their eyes from the beginning of the encoding phase, to the end of the recall phase: thus creating a proprioception-only task.

\section{Insert Figure 4 here}

Experimental trial blocks constituted familiarisation (15 trials), acquisition 1 (30 trials), transfer 1 (30 trials), acquisition 2 (30 trials) and transfer 2 (30 trials). Familiarisation and acquisition targets were 122,132 and $142^{\circ}$. In transfer, participants were given new targets, which were 117,127 and $147^{\circ}$. Participants were informed of this. Target order was randomised between participants and trial blocks: with the stipulation that targets were never repeated immediately. At familiarisation, participants were given no focus of attention instructions. At acquisition 1, half of participants were given external focus of attention instructions ("focus on the markers, and how you move them to their correct position") and the other half internal focus of attention instructions ("focus on your leg, and move it to its correct position"). At acquisition 2, participants were given the focus of attention instructions they did not receive in acquisition 1. It was highlighted to 
1 participants that it was very important for them to stick to their prescribed focus of attention. At acquisition, focus reminders were provided at every trial for the first five trials, after which they were given every 10 trials. At transfer, participants were given no reminders. As a manipulation check, after every block of trials, participants were given a piece of paper to write down what they focused on in their block of trials; participants were asked to provide sufficient detail for someone else to replicate their focus. In summary the present study design entailed a within-group trial block factor and counterbalanced within-group focus of attention factor for weight and no-weight groups.

\section{Data reduction and dependent measures.}

During testing, a custom Microsoft Visual Basic two-point central finite difference algorithm was used to calculate instantaneous velocity data from displacement data. This process was repeated to calculate acceleration data from velocity data. Movement initiation was defined as the data point where velocity dropped to $<1$ degree per second. Movement end was defined as one data point before leg velocity reached $\leq 0$ : meaning movements could not have a reversal in movement direction. MT for participants' post-trial feedback was defined as the time (ms) between movement initiation and movement end. Recall phase error for participants' feedback was defined as the angular deviation between leg angle at movement end and the target position; overshoots were labelled numerically positive and undershoots numerically negative. For post-testing analysis, using a custom LabView script, leg displacement data was filtered using a second-order dual-pass Butterworth filter with a $10 \mathrm{~Hz}$ low-pass cut-off frequency. Movement start, end and time were recalculated using this filtered data. Additionally, four kinematic markers were calculated using custom LabView scripts. Namely, peak acceleration (pka), peak velocity (pkv), peak negative acceleration (pkna) and movement end (end). Encoding error for analysis was calculated as the angular deviation between movement end and the encoded position. Overshoots were labelled numerically positive and undershoots numerically negative. 
Trials whose angular movement distance deviated by $>$ 2SD from the given participant's trial

block mean for $>25 \%$ of the movement distance were removed prior to analysis $(2.07 \%$ of all trials). Data of participants who did not change their focus $(n=5)$ between acquisition 1 and 2, based on two researchers' impression of the manipulation check responses, were also removed from analysis (see below examples and resulting group sizes ${ }^{3}$ ). Dependent measures were based on LabView-filtered data and included: MT; encoding CE (mean within-participant encoding error); and encoding VE (standard deviation of within-participant encoding error). Spatial variability throughout the amplitude of movement was assessed via variability in movement degrees at each kinematic marker (i.e., pka, pkv, pkna, and end). There was no directional component to the task. Vastus medialis and vastus lateralis EMG activity (in V) for each trial was analysed offline from 20ms before movement start until movement end: using custom MATLAB scripts (MathWorks Inc., Natick, MA). EMG activity was filtered using a bidirectional fourth order bandpass (20-450hz) Butterworth filter, rectified, and integrated to attain a measure of muscular activation within the context of time (i.e., iEMG).

\section{Analysis.}

For brevity and clarity, the analysis on endpoint CE, endpoint AE, endpoint VE, and VE at each kinematic marker, used data collapsed across targets; separate 3 Target (low, medium, and high) x 2 Trial Block (acquisition and transfer) x 2 Focus of Attention (internal and external) ANOVAs for each weight group were performed on endpoint CE, AE, and VE to ensure collapsing targets was appropriate and revealed no significant interactions involving targets. To ensure the counterbalanced subsets of participants did not differ at the beginning of testing, familiarisation iEMG and familiarisation endpoint MT, CE, AE, and VE were submitted to independent samples t-

\footnotetext{
${ }^{3}$ Examples statements that suggested participants did not adhere to the appropriate focus: an external focus that was reported as focusing on "using muscle memory based on how tense the leg muscle is", "leg speed and trying to keep my leg still" or comments which suggested the participant was using neither an internal nor external focus e.g. "my mind was more neutral", "I wasn't really focused on a specific thing". Post data-reduction n=35 (No-weight external 1st then internal $=10$; No-weight internal 1 st then external $=8$; Weight external 1 st then internal $=7$; Weight internal 1 st then external $=10$ ).
} 
tests which compared external-to-internal and internal-to-external focus of attention subgroups, within each weight condition (i.e. two comparisons per-dependent variable). To investigate focus of attention's effect on muscular efficiency and outcome performance, iEMG of the vastus lateralis and iEMG of the vastus medialis were then analysed using separate 2 Weight (weighted and noweight) x 2 Trial Block (acquisition and transfer) x 2 Order (external-to-internal and internal-toexternal) x 2 Focus of Attention (internal \& external) ANOVAs. The effect of focus of attention on offline planning was investigated via separate 2 Trial Block (acquisition and transfer) $\mathrm{x} 2$ Focus of Attention (internal and external) 2 Order (external-to-internal and internal-to-external) x 2 Marker (pka and pkv) ANOVAs for each weight group (weighted and no-weight). The effect of focus of attention on online control was investigated via separate 2 Trial Block (acquisition and transfer) x 2 Focus of Attention (internal and external) x 2 Order (external-to-internal and internal-to-external) $\mathrm{x}$ 3 Marker (pkv, pkna, and end) ANOVA for each weight group (weighted and no-weight). The purpose of the order factor was to determine whether switching from an internal to an external focus of attention, or vice versa, impacted performance. The assumption for sphericity held in all analyses since no more than two levels of within-subject factors were compared.

\section{Results}

\section{Test of equivalence for counterbalanced subsets}

T-tests comparing familiarisation CE of external-to-internal and internal-to-external focus of attention subgroups within weight and no-weight groups revealed no significant difference in the no-weight group $(t(16)=-.45, p=.659,95 \% \mathrm{CI}[-4.83,3.14]$ nor the weight group $(t(15)=1.06, p$ $=.306,95 \% \mathrm{CE}[-1.52,4.52]) . \mathrm{T}$-tests comparing familiarisation $\mathrm{AE}$ of external-to-internal and internal-to-external subgroups within weight and no-weight groups revealed no significant difference in the no-weight group $(t(16)=-.79, p=.444,95 \% \mathrm{CI}[-4.37,2.01]$ nor the weight group $(t(15)=1.07, p=.302,95 \% \mathrm{CE}[-1.12,3.38])$. T-tests comparing familiarisation VE of external-tointernal and internal-to-external focus of attention subgroups within weight and no-weight groups 
revealed no significant difference in the no-weight group $(t(16)=-1.53, p=.144,95 \% \mathrm{CI}[-1.39$, $.22])$ nor the weight group $(t(15)=1.29, p=.216,95 \% \mathrm{CE}[-.31,1.25])$. The T-tests comparing familiarisation iEMG of external-to-internal and internal-to-external focus of attention subgroups within weight $(t(16)=-.17, p=.869,95 \% \mathrm{CE}[-.04, .03] ; t(16)=.64, p=.533,95 \% \mathrm{CE}[-.01, .03]$, respectively for vastus medialis and vastus lateralis) and no-weight $(t(15)=1.41, p=.178,95 \% \mathrm{CE}$ $[-.01, .06] ; t(16)=-.10, p=.924,95 \% \mathrm{CE}[-.03, .03]$, respectively for vastus medialis and vastus lateralis) groups revealed no significant difference and therefore subgroups should be comparable.

\section{Outcome performance.}

Results of the 2 Focus of Attention x 2 Trial Block x 2 Order ANOVAs on weight and noweight groups' CE, AE, and VE are shown in table 3B. Analysis of the no-weight group CE revealed a near-significant Trial Block x Focus of Attention x Order interaction (see figure 5, panels A, C, and E) and a significant main effect for trial block: with no other significant main effects nor interactions. The main effect for trial block revealed acquisition generally featured significantly less $\mathrm{CE}$ than transfer. Analysis of the weighted group CE revealed a near-significant focus of attention main effect and significant Focus of Attention x Order interaction (see figure 5, panels B, D and F): with no other significant main effects nor interactions. Specifically, when weighted participants adopted an internal focus towards the end of testing, their CE was significantly reduced. Analysis of the no-weight group AE revealed only a significant main effect for trial block: wherein acquisition generally featured significantly less AE than transfer. Analysis of the weighted group AE revealed no significant main effects nor interactions. Analysis of the no-weight group VE revealed only a significant trial block main effect: wherein acquisition generally featured significantly less VE than transfer. Analysis of the weighted group VE revealed no significant main effects nor interactions.

\section{Offline planning / online control contributions to outcome performance.}

Results of the 2 Trial Block x 2 Focus of Attention x 2 Marker ANOVAs performed on the weight and no-weight groups to infer offline planning efficacy are shown in Table 3D. Analysis of 
the no-weight group revealed only significant main effects for trial block and marker and a significant Focus of Attention x Trial Block interaction. Breakdown of the Focus of Attention $\mathrm{x}$ Trial Block x Order interaction revealed that when an internal focus of attention came second, variability was reduced. Analysis of the weighted group revealed significant main effects for trial block and marker and a significant Focus of Attention x Trial Block x Marker x Order interaction. Breakdown of this interaction also revealed that when an internal focus of attention came second, variability was reduced but only in acquisition and at peak velocity (by which point differences in planning should be at their most prominent). Results of the 2 Trial Block x 2 Focus of Attention x 2 Marker ANOVAs performed on the weight and no-weight groups to infer online control efficacy are shown in Table 3D. Analysis of neither the no-weight group nor the weighted group revealed any significant interactions involving markers. In sum, these results suggest that differences in outcome performance based on focus of attention are primarily facilitated via changes in planning.

\section{Insert Table 3 here}

\section{Muscle activity.}

The 2 Focus of Attention x 2 Trial Block x 2 Weight x Order ANOVA on the vastus lateralis iEMG revealed a significant main effect for focus of attention $\left(F_{1,31}=4.86, p=.035, \eta_{\mathrm{p}}^{2}=\right.$ $.14)$ and Focus of Attention $\mathrm{x}$ Weight $\mathrm{x}$ Order interaction $\left(F_{1,31}=8.06, p=.008, \eta_{\mathrm{p}}{ }^{2}=.21\right)$. Specifically, an internal $(\mathrm{M}=.058, \mathrm{SD}=.029)$ generally yielded lower iEMG compared to an external focus $(\mathrm{M}=.060, \mathrm{SD}=.024)$ and the Focus of Attention $\mathrm{x}$ Weight $\mathrm{x}$ Order interaction followed the same pattern of results as CE. The 2 Focus of Attention x 2 Trial Block $x 2$ Weight $x$ Order ANOVA on the vastus medialis iEMG revealed a main effect for focus of attention and Focus of Attention $\mathrm{x}$ Weight $\mathrm{x}$ Order interaction which trended in the same direction (as the vastus laterialis data) but were not significant $\left(F_{1,31}=2.92, p=.097, \eta_{\mathrm{p}}^{2}=.09 ; F_{1,31}=3.35, p=.077, \eta_{\mathrm{p}}{ }^{2}=\right.$ .10 , respectively). 


\section{Discussion}

Experiment 3 utilised an amplitude-only leg extension task based on that of Toussaint et al. (2016). Participants were tasked with encoding target leg positions and accurately reproducing them in one fast target-directed movement, under counterbalanced external and internal focus of attention instructions. We aimed to ascertain whether, contrary to the assertions of the constrained action hypothesis (Wulf et al., 2001), an internal focus of attention can facilitate superior performance: specifically, when proprioceptive feedback strength was increased via an ankle weight. It was hypothesised that, compared to an external focus of attention, an internal focus would facilitate superior performance, because it guides participants' attention to task-relevant proprioceptive information. These external / internal focus of attention differences were hypothesised to be stronger in the group performing with an ankle weight.

Outcome performance was inferred from overall endpoint CE, AE, and VE. Results revealed that for the weighted group, participants who adopted an internal focus later in testing, reduced their CE during acquisition trials. Furthermore, the weighted group's CE exhibited a near-significant focus of attention main effect $(p=.052)$ and a significant focus of attention by marker interaction: wherein adopting an internal focus reduced CE compared to an external focus. Specifically, an internal focus significantly reduced CE when it was the second focus participants were given (i.e., later in testing, after an external focus). These effects were not significant for the weighted group's AE, but followed the same trends as per their CE. Concerning the weighted group's endpoint VE, there was no difference between external and internal groups. Past studies may help us understand the mechanisms behind our observed focus of attention order effects (McNevin, Shea, \& Wulf, 2003; Wulf et al., 1998). Focus of attention effects in novices seemingly manifest during later testing phases. Therefore, in Experiment 3, an internal focus may have been more beneficial when provided following an 
external focus because by that point, it could be argued that participants were more accustomed to utilising the proprioceptive feedback of the task. There is a body of literature suggesting enhanced proprioceptive neural mechanisms over time through neuroplasticity as a function of practice / dependency (Schwenkreis, Pleger, Höffken, Malin \& Tegenthoff. 2001; Goble, 2010; Xerri, 2012).

In sum, the endpoint results of Experiment 3 demonstrate that an external focus does not always facilitate 'best' performance. Instead the improved CE and (although non-significantly) improved AE, combined with decreases in VE at pkv, suggest that an internal focus overall facilitated superior outcome performance over an external focus when the task was reliant on proprioception and proprioceptive strength was high (i.e. within the weight group). The internal focus' performance superiority seemingly originated within offline movement planning based on decreased variability at key kinematic markers attributable to planning mechanisms (i.e., pkv) for those adopting an internal focus. Concurrently, an internal focus yielded less EMG compared to an external focus, suggesting that adopting an internal focus facilitates more efficient / appropriate planning of muscle activation when tasks are proprioception-based. Overall, Experiment 3 lends further credence to the notion that, contrary to the constrained action hypothesis of Wulf et al. (2001), an internal focus can be superior to an external focus: provided proprioceptive strength and pertinence is high.

Analyses were conducted to ensure the aforementioned performance differences were due to focus of attention instructions and not confounds. Focus of attention effects were investigated using a repeated measures design and data analysis considered whether focus of attention order (i.e., external focus 1 st and internal focus 2 nd, or vice versa) influenced findings. To ensure the externalto-internal and internal-to-external subsets of participants within each weight group were comparable, independent samples t-tests compared these subsets' endpoint MT, CE, AE, and VE at familiarisation (i.e., the beginning of testing where participants were not yet prescribed a focus of attention). This analysis revealed no significant differences.

\section{General Discussion}


The last decade has seen considerable advances in our understanding of the more pertinent characteristics in formulating effective instruction. Endorsement of externally focused and implicit information in promoting automaticity of movements seems well grounded in the literature, with mechanisms such as Prinz's (1997) 'action-effect principle' and Wulf et al.'s (2001) 'constrained action hypothesis' conveying similar messages. Nevertheless, this theoretical understanding has failed to align with mechanisms accounting for the benefits of process goals and external/kinaesthetic motor imagery, both of which encourage attentional shifts towards and not away from movements. The current investigation has attempted to begin to address this research lacuna, over three experiments designed to identify nuances where an internal focus may be useful. This is in line with a proposed facilitative 'somaesthetic awareness' (Shusterman, 2011; Toner \& Moran, 2015) when making error corrections or re-learning movements.

In essence, it has not yet been specifically tested whether it is changes in offline planning, online movement adjustments, or both forms of motor control that facilitate improved outcome performance with an external focus of attention, nor whether an internal focus of attention also has the capability to enhance components of motor control / outcome performance when pertinent task information is of proprioceptive (i.e. internal) nature. A noteworthy effort to partially address the former research lacuna was by Lohse and colleagues $(2010 ; 2012)$. Two studies respectively utilised time between trials and pre-movement time to infer offline movement planning efficiency. In line with the conjecturing of the constrained action hypothesis (Wulf et al., 2001), both studies demonstrated that an external focus of attention facilitated improved force-production accuracy, whilst concurrently reducing the time used for offline planning: indicating reduced conscious processing and increased automaticity compared to an internal focus of attention. However, two limitations of these studies are that, firstly, time used for offline planning was a measure of efficiency (i.e., how many cognitive resources were utilised) rather than efficacy (i.e., how good the movement plan was) and secondly, online motor control's role within performance was not explicitly measured. 
1 Therefore, offline planning and/or online motor control's precise contribution to the creation of outcome performance under different foci of attention is unknown. Our first experiment aimed to address this question via a fast target-directed visual aiming task, wherein the variability methodology of Khan et al. (2006) was used to simultaneously infer offline planning and online motor control's contribution to outcome performance.

The results of Experiment 1 revealed no differences between foci in the directional component of movement, but within the amplitude component, the internal group displayed reduced (i.e. superior) movement endpoint $\mathrm{CE}, \mathrm{AE}$, and $\mathrm{VE}$ compared to the external group. In sum, participants exhibited superior outcome performance when an internal focus was adopted. Variability findings confirmed that focus of attention-based differences manifest within offline movement planning. The internal group demonstrated overall reduced variability throughout movement compared to the external group, but the shape of these variability profiles did not differ. The underlying processes yielding Experiment 1's results are likely several fold. Firstly, the fine motor skill nature of our visualaiming task likely raised the pertinence of proprioceptive information. The internal group may have been able to exploit the congruent (i.e., internal in nature) feedback of the task and achieve better performance than the external group via improved movement planning. Secondly, differences may have manifested within the amplitude component of movement because the impulse and resultant proprioceptive feedback generated to move the hand an amplitude of $20 \mathrm{~cm}$, to the target, should be exponentially greater than any impulse / feedback generated within the directional component.

Experiment 2 and 3 aimed to further Experiment 1 by increasing the congruence between pertinent task characteristics and participants' internal focus of attention. Experiment 2 removed vision during the aiming task to increase proprioceptive task demands (i.e., participants had to identify target locations through proprioception only). In line with our hypotheses, results revealed that an internal focus overall yielded superior outcome performance within the amplitude and direction components of movement. Analysis of variability throughout movement lent further credence to the 
notion that focus of attention effects originate within offline movement planning for non-continuous tasks. As in Experiment 1, movement variability was reduced with an internal compared to an external focus, but the shape of variability profiles did not differ. Finally, Experiment 3 aimed to build on Experiment 2 via the utilisation of a leg-extension task and the modulation of proprioceptive feedback strength: via a weight attached to a single group of participants' ankles. The provision of an ankle weight should increase proprioceptive feedback strength by increasing musculotendinous mechanoreceptor sensitivity (Bullock-Saxton, Wong, \& Hogan, 2001; Suprak, Ostering, Donkelaar, \& Karduna, 2007). Results within the no-weight group demonstrated significantly lower $(p=0.05)$ demonstrated less endpoint $\mathrm{CE}$ when adopting an internal compared to an external focus.

Concurrently, across both weight-groups, an internal focus yielded more efficient muscle activation compared to an external focus, and the shape of internal and external variability profiles were again indicative of enhanced planning mechanisms under an internal focus.

In sum, our findings provide strong support to the notion that focus of attention-based performance differences originate within offline movement planning for non-continuous tasks. Additionally, our results support the notion of facilitative somaesthetic awareness and that an internal focus congruent with task demands can benefit performance and efficiency. This finding makes logical sense when one considers an external focus also has to be congruent with the intended external movement outcome to facilitate optimal performance (e.g. focusing on the dart-board when the task requires the dart-board to be hit) (Land, Tenenbaum, Ward, \& Marquardt, 2013; Russell, Porter, \& Campbell, 2014). Similarly, Pelleck and Passmore (2017) show that when novice golfers adopted an internal focus more closely related to a putting task, where there was a clear external outcome demand and proprioceptive salience was arguably low, a number of performance variables suffered including accuracy, EMG and movement kinematics. It is possible that had the external focus direction within experiment 1 and 2 been directed towards the target as opposed to the cursor itself, task demands may 
1 have been more congruent with an external focus and thus, it could be argued that we would have seen a benefit of an external focus in this instance. However, the authors wanted to maintain comparability between internal and external instructions to avoid a confound as per recommendations from Wulf (2013).

However, it should be noted that the current study is not the first to investigate focus of attention effects within tasks featuring high proprioceptive demands, but is the first with the explicit aim of investigating internal focus of attention and task congruence. Limitations of prior studies which prohibit the generalisation of their results to this research question include: task instructions muddling an internal and external focus (Lohse et al., 2011; Lohse, 2012); internal focus instructions that may not have been congruent with the external task demands / may only have directed attention to a small subset of task-relevant proprioception (Land et al., 2013; Makaruk, Porter, \& Makaruk, 2013; Sherwood, Lohse, \& Healy, 2014); and tasks which were primarily dependent on online motor corrections (Schlesinger, Porter, \& Russell, 2013). It is important to highlight that despite proprioception's arguably heightened pertinence in force production tasks, prior studies adopting this approach have shown some converse results to that of the current research in target-directed aiming tasks (e.g. Freedman, Caligiuri, Wulf, \& Robin, 2007). We argue that the simplistic nature of these tasks is a likely contributor to this. In these instances, task simplicity would have meant reduced availability of proprioceptive information to enhance planning and thus, no benefits from an internal focus.

\section{Conclusion.}

Theoretical implications derived from attentional focus literature advocate a perpetual external focus for optimal movement execution, but this is incompatible with promotion of widely used process goals and external/kinaesthetic imagery for practitioners. The current findings refute the rigidity of the constrained action hypothesis and provide evidence that an internal focus can facilitate superior performance and efficiency over an external focus when congruent with task 
1 demands. This has strong implications for sports or tasks where proprioceptive salience is high, such as diving, artistic gymnastics or weightlifting. Where traditional attentional focus literature would advocate external instructions and feedback within these tasks (see Wulf, 2013 for a review) e.g. a focus towards the surface of the water, support surface or bar respectively, the current findings would suggest a focus on the body movements themselves. In line with the principles underlying specificity theory (Proteau, 1992), sources of afferent information most useful to performance execution are typically prioritised during processing. Thus, adopting an internal focus within these sports should enhance congruence between the 'instructions and feedback provided' and the 'availability of pertinent afferent information for task execution', which is largely proprioceptive in nature within these sports (see Figure 6 for sporting examples with varying degrees of pertinence of proprioceptive and visual information for task execution). We suggest internal instructions and feedback for tasks high in pertinence of proprioceptive information but low in pertinence of visual information and external instructions and feedback for tasks low in pertinence of proprioceptive information but high in pertinence of visual information. With this in mind, it is therefore not a surprise that there seems more robust evidence for benefits of an external compared to internal focus when most sports / tasks rely heavily on visual information for task success (e.g. towards a ball or target). Clearly not all tasks can be classified as exclusively high or low in pertinence of proprioceptive and visual information for task execution. Thus, we would suggest that this works on a continuum basis with some sports likely benefitting from both forms of instructions and feedback in different situations. This is in line with aforementioned arguments made by Collins et al. (2015) and Lawrence et al. (2011) discussing nuances of attentional focus instructions dependent on instructional familiarisation, task relevance and salience of movement effects for task execution.

The present experiments also provide compelling evidence that attentional focus effects manifest within offline movement planning for non-continuous tasks. This may have practical 
1 implications for performance under pressure where athletes are more likely to adopt strategies (such

2 as an internal focus in proprioceptive tasks) to improve movement planning as a reaction to pressure

3 inhibiting the effectiveness of error corrections made during a movement (see Allsop, Lawrence,

4 Gray \& Khan, 2017). By focusing on the body, findings suggest a shift in importance to offline

5 planning processes, which subsequently should also inhibit any decrements in response

6 programming under pressure.

7

8

Insert Figure 6 here

9

10 
References

Allsop, J.E., Lawrence, G.P., Gray, R., \& Khan, M.A. (2017). The interaction between practice and performance pressure on the planning and control of fast target directed movement. Psychological Research, 81, 1004-1019. doi: 10.1007/s00426-016-0791-0

Alkner, B. A., Tesch, P. A., \& Berg, H. E. (2000). Quadriceps EMG/force relationship in knee extension and leg press. Medicine and Science in Sports and Exercise, 32, 459-463. doi: 10.1097/00005768-200002000-00030

Beilock, S.L., Carr, T.H., MacMahon, C., \& Starkes, J.L. (2002). When paying attention becomes counterproductive: impact of divided versus skill-focused attention on novice and experienced performance of sensorimotor skills. Journal of Experimental Psychology: Applied, 8, 6-16. http://dx.doi.org/10.1037/1076-898X.8.1.6

Bernstein, N. (1967). The co-ordination and regulation of movement. London: Pergamon Press.

Bullock-Saxton, J. E., Wong, W. J., \& Hogan, N. (2001). The influence of age on weight-bearing joint reposition sense of the knee. Experimental Brain Research, 136, 400-406. doi $10.1007 / \mathrm{s} 002210000595$

Carlton, L.G. (1992). Visual processing time and the control of movement. In L. Proteau \& D. Elliott (Eds.), vision and motor control (pp. 3-31). North-Holland: Amsterdam.

Chua, R., \& Elliott, D. (1993). Visual regulation of manual aiming. Human Movement Science, 12, $365-401$. https://doi.org/10.1016/0167-9457(93)90026-L

Collins, D., Carson, H.J., \& Toner, J. (2016). Letter to the editor concerning the article "Performance of gymnastics skill benefits from an external focus of attention" by Abdollahipour, Wulf, Psotta \& Nieto (2015). Journal of Sports Sciences, 34, 1288-1292. 
Examining the specificity of practice hypothesis: is learning modality specific? Research Quarterly

Crossman, E.R., \& Goodeve, P.J. (1983). Feedback control of hand-movement and Fitts' Law. Quarterly Journal of Experimental Psychology, 35, 251-78. https://doi.org/10.1080/14640748308402133

Elliott, D., Chua, R., Pollock, B.J., \& Lyons, J. (1995). Optimizing the use of vision in manual aiming: the role of practice. The Quarterly Journal of Experimental Psychology Section A, $48,72-83$.

doi: 10.1080/14640749508401376

Elliott, D., Dutoy, C., Andrew, M., Burkitt, J.J., Grierson, L.E.M., Lyons, J.L., Hayes, S.J., \& Bennett, S.J. (2014). The influence of visual feedback and prior knowledge about feedback on vertical aiming strategies. Journal of Motor Behavior, 46, 433-443. doi: $10.1080 / 00222895.2014 .933767$

Elliott, D., Helsen, W.F., \& Chua, R. (2001). A Century Later: Woodworth's (1899) TwoComponent Model of Goal-Directed Aiming. Psychological Bulletin, 127, 342-357. doi: 10.1037//0033-2909.127.3.342

Ericsson, K.A., Krampe, R.T. \& Tesche-Römer, C. (1993). The role of deliberate practice in the acquisition of expert performance. Psychological Review, 100, 363-406. doi:10.1037/0033-295X.100.3.363

Faul, F., Erdfelder, E., Lang, A.G., \& Buchner, A. (2007). G*Power 3: a flexible statistical power analysis program for the social, behavioral, and biomedical sciences. Behavior Research Methods, 39, 175-191. https://doi.org/10.3758/BF03193146 
Freedman, S. E., Maas, E., Caligiuri, M. P., Wulf, G., \& Robin, D. A. (2007). Internal versus external: oral motor performance as a function of attentional focus. Journal of Speech, Language and Hearing Research, 50, 131-136. doi:10.1044/1092-4388(2007/011)

Goble D.J. (2010). Proprioceptive acuity assessment via joint position matching: from basic science to general practice. Physical Therapy, 90, 1176-1184. https://doi.org/10.2522/ptj.20090399

Hardy, L., \& Callow, N. (1999). Efficacy of External and Internal Visual Imagery Perspectives for the Enhancement of Performance on Tasks in Which Form Is Important. Journal of Sport and Exercise Psychology, 21, 95-112. https://doi.org/10.1123/jsep.21.2.95

Hermens, H. J., Freriks, B., Merletti, R., Stegeman, D., Blok, J., Rau, G., ... \& Hägg, G. (1999). European recommendations for surface electromyography. Roessingh Research and Development, 8, 13-54. Retrieved from http://www.seniam.org/pdf/contents8.PDF

Khan, M. A., Elliott, D., Coull, J., Chua, R., \& Lyons, J. (2002). Optimal control strategies under different feedback schedules: Kinematic evidence. Journal of Motor Behavior, 34, 45-57. https://doi.org/10.1080/00222890209601930

Khan, M.A, Franks, I.M., Elliott, D., Lawrence, G.P., Chua, R., Bernier, P., Hansen, S., \& Weeks, D.J. (2006). Inferring online and offline processing of visual feedback in target-directed movements from kinematic data. Neuroscience and Biobehavioral Reviews, 30, 11061121. doi:10.1016/j.neubiorev.2006.05.002

Khan, M.A., Lawrence, G.P., Fourkas, A., Franks, I.M., Elliott, D., \& Pembroke, S. (2003). Online versus offline processing of visual feedback in the production of movement distance. Acta Psychologica, 113, 83-97. https://doi.org/10.1016/S0001-6918(02)00156-7 
1 Land, W. M., Tenenbaum, G., Ward, P., \& Marquardt, C. (2013). Examination of visual information as a mediator of external focus benefits. Journal of Sport and Exercise Psychology, 35, 250-259. https://doi.org/10.1123/jsep.35.3.250

Lawrence, G.P., Gottwald, V.M., Hardy, J., \& Khan, M.A. (2011). Internal and external focus of attention in a novice form sport. Research Quarterly for Exercise and Sport, 82, 431-441. doi: 10.1080/02701367.2011.10599775

Lohse, K.R. (2012). The influence of attention on learning and performance: pre-movement time and accuracy in an isometric force production task. Human Movement Science, 31, 12-25. doi:10.1016/j.humov.2011.06.001

Lohse, K., Sherwood, D., \& Healy, A. (2010). How changing the focus of attention affects performance, kinematics, and electromyography in dart throwing. Human Movement Science, 29, 542-555. doi:10.1016/j.humov.2010.05.001

Lohse, K. R., Sherwood, D. E., \& Healy, A. F. (2011). Neuromuscular effects of shifting the focus of attention in a simple force production task. Journal of Motor Behavior, 43, 173-184. https://doi.org/10.1080/00222895.2011.555436

Makaruk, H., Porter, J. M., \& Makaruk, B. (2013). Acute effects of attentional focus on shot put performance in elite athletes. Kinesiology: International Journal of Fundamental and Applied Kinesiology, 45, 55-62. doi: $796.433 .1: 796.015 .15$

McNevin, N.H., Shea, C.H., \& Wulf, G. (2003). Increasing the distance of an external focus of attention enhances learning. Psychological Research, 67, 22-29. doi:10.1007/s00426-002-0093-6 
1 Pelleck, V., \& Passmore, S.R. (2017). Location versus task relevance: The impact of differing internal focus of attention instructions on motor performance. Acta Psychologica, 176, 23 31. https://doi.org/10.1016/j.actpsy.2017.03.007

Porter, J.M., Wu, W.F.W., \& Partridge, J.A. (2010). Focus of attention and Verbal Instructions: Strategies of Elite Track and Field Coaches and Athletes. Sport Science Review, XIX, 77-89. doi:10.2478/v10237-011-0018-7

Prinz, W. (1997). Perception and action planning. European Journal of Cognitive Psychology, 9, 129-154. https://doi.org/10.1080/713752551

Proteau, L. (1992). On the specificity of learning and the role of visual information for movement control. In L. Proteau \& D. Elliott (Eds.), Advances in psychology, No. 85. Vision and motor control (pp. 67-103). Oxford, England: North-Holland. http://dx.doi.org/10.1016/S0166-4115(08)62011-7

Russell, R., Porter, J., \& Campbell, O. (2014). An external skill focus is necessary to enhance performance. Journal of Motor Learning and Development, 2, 37-46. https://doi.org/10.1123/jmld.2014-0038

Schlesinger, M., Porter, J., \& Russell, R. (2013). An external focus of attention enhances manual tracking of occluded and visible targets. Frontiers in Psychology, 3, 591. https://doi.org/10.3389/fpsyg.2012.00591

Schmidt, R. A., Zelaznik, H., Hawkins, B., Frank, J. S., \& Quinn Jr, J. T. (1979). Motor-output variability: a theory for the accuracy of rapid motor acts. Psychological Review, 86, 415. http://dx.doi.org/10.1037/0033-295X.86.5.415 
Schwenkreis P., Pleger B., Höffken O., Malin J.P., \& Tegenthoff M. (2001). Repetitive training of a synchronised movement induces short-term plastic changes in the human primary somatosensory cortex. Neuroscience Letters, 312, 99-102. https://doi.org/10.1016/S0304-3940(01)02196-6

Sherwood, D. E., Lohse, K. R., \& Healy, A. F. (2014). Judging joint angles and movement outcome: Shifting the focus of attention in dart-throwing. Journal of Experimental Psychology: Human Perception and Performance, 40, 1903. doi: $10.1037 / \mathrm{a} 0037187$

Shusterman, R. (2011). “Somaesthetic Awareness, Proprioceptive Perception, and Performance," in Consciousness, Perception, and Behavior: Conceptual, Theoretical, and Methodological Issues, Proceedings of the 11th Biannual Symposium on the Science of Behavior, Guadalajara, México, February 2010, E. R. Iñesta and J. E. Burgos (eds.), New Orleans: University Press of the South.

Suprak, D. N., Osternig, L. R., van Donkelaar, P., \& Karduna, A. R. (2007). Shoulder joint position sense improves with external load. Journal of Motor Behavior, 39, 517-525. https://doi.org/10.3200/JMBR.39.6.517-525

Toner, J., \& Moran, A. (2015). Enhancing performance proficiency at the expert level: Considering the role of 'somaesthetic awareness'. Psychology of Sport and Exercise, 16, 110-117. https://doi.org/10.1016/j.psychsport.2014.07.006

Toner, J., \& Moran, A. (2016). On the importance of critical thinking: A response to Wulf's (2015) commentary. Psychology of Sport and Exercise, 22, 339-340. https://doi.org/10.1016/j.psychsport.2015.05.007

Toussaint, L., \& Blandin, Y. (2010). On the role of imagery modalities on motor learning. Journal of Sports Sciences, 28, 497-504. https://doi.org/10.1080/02640410903555855 
1 Toussaint, L., Meugnot, A., Badets, A., Chesnet, D., \& Proteau, L. (2016). The specificity of practice hypothesis in goal-directed movements: visual dominance or proprioception neglect? Psychological Research, 81, 407-414. https://doi.org/10.1007/s00426-016-0748-3

Willingham, D.B. (1998). A neuropsychological theory of motor learning. Psychological Review, $105,558-584$. doi:10.1037/0033-295X.105.3.558

Wolpert, D. M., \& Kawato, M. (1998). Multiple paired forward and inverse models for motor control. Neural Networks, 11, 1317-1329. https://doi.org/10.1016/S0893-6080(98)00066-5

Woodworth, R. S. (1899). Accuracy of voluntary movement. The Psychological Review: Monograph Supplements, 3, i. http://dx.doi.org/10.1037/h0092992

Wulf, G. (2013). Attentional focus and motor learning: a review of 15 years. International Review of Sport and Exercise Psychology, 6, 77-104. https://doi.org/10.1080/1750984X.2012.723728

Wulf, G. (2015). Why did Tiger Woods shoot 82? A commentary on Toner and Moran (2015). Psychology of Sport and Exercise, 22, 337-338. https://doi.org/10.1016/j.psychsport.2015.05.006

Wulf, G., Höß, M., \& Prinz, W. (1998). Instructions for motor learning. Differential effects of internal versus external focus of attention. Journal of Motor Behaviour, 30, 169-179. https://doi.org/10.1080/00222899809601334

Wulf, G., McNevin, N.H., \& Shea, C.H. (2001). The automaticity of complex motor skill learning as a function of attentional focus. Quarterly Journal of Experimental Psychology, 54, 11431154.

https://doi.org/10.1080/713756012 
1 Xerri C. (2012). Plasticity of cortical maps: multiple triggers for adaptive reorganization following brain damage and spinal cord injury. Neuroscientist, 18, 133-148. https://doi.org/10.1177/1073858410397894

4

Zimmerman, B. J., \& Kitsantas, A. (1997). Developmental phases in self-regulation: Shifting from process goals to outcome goals. Journal of Educational Psychology, 89, 29-36.

6 http://dx.doi.org/10.1037/0022-0663.89.1.29

7

8

9

10

11 


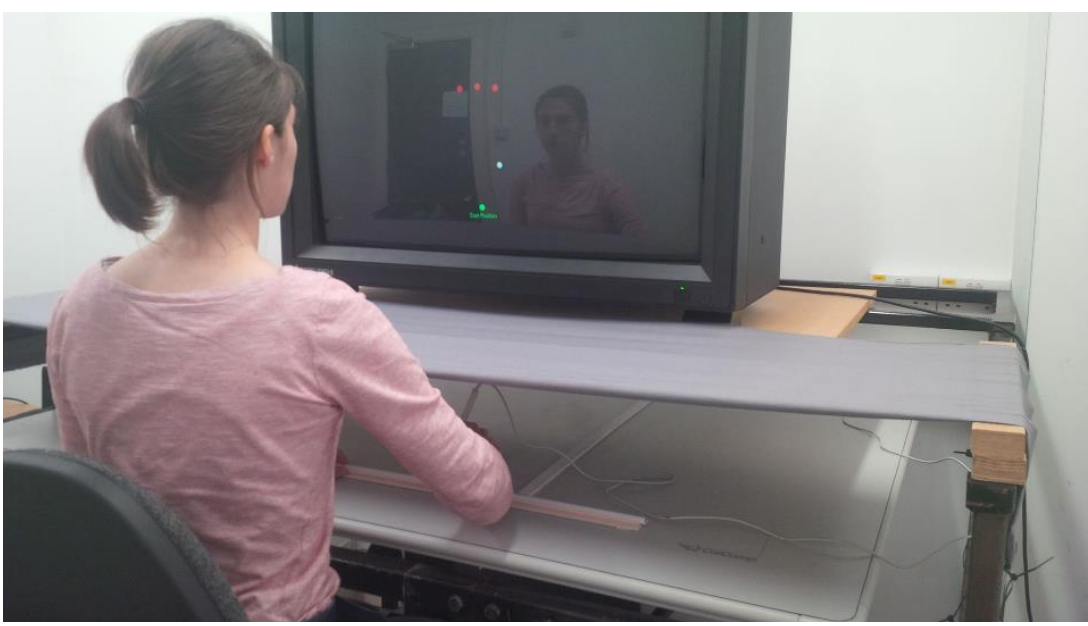

Figure 1. Experiment 1. The task was a target-directed aiming movement, performed using a handheld stylus on a digitising tablet whereby the $\mathrm{X} / \mathrm{Y}$ movement of the stylus on the tablet plane corresponded to the X/Y movement of the cursor on the monitor plane. Participants were required to move the cursor from the start position to one of three targets as quickly and accurately as possible. 


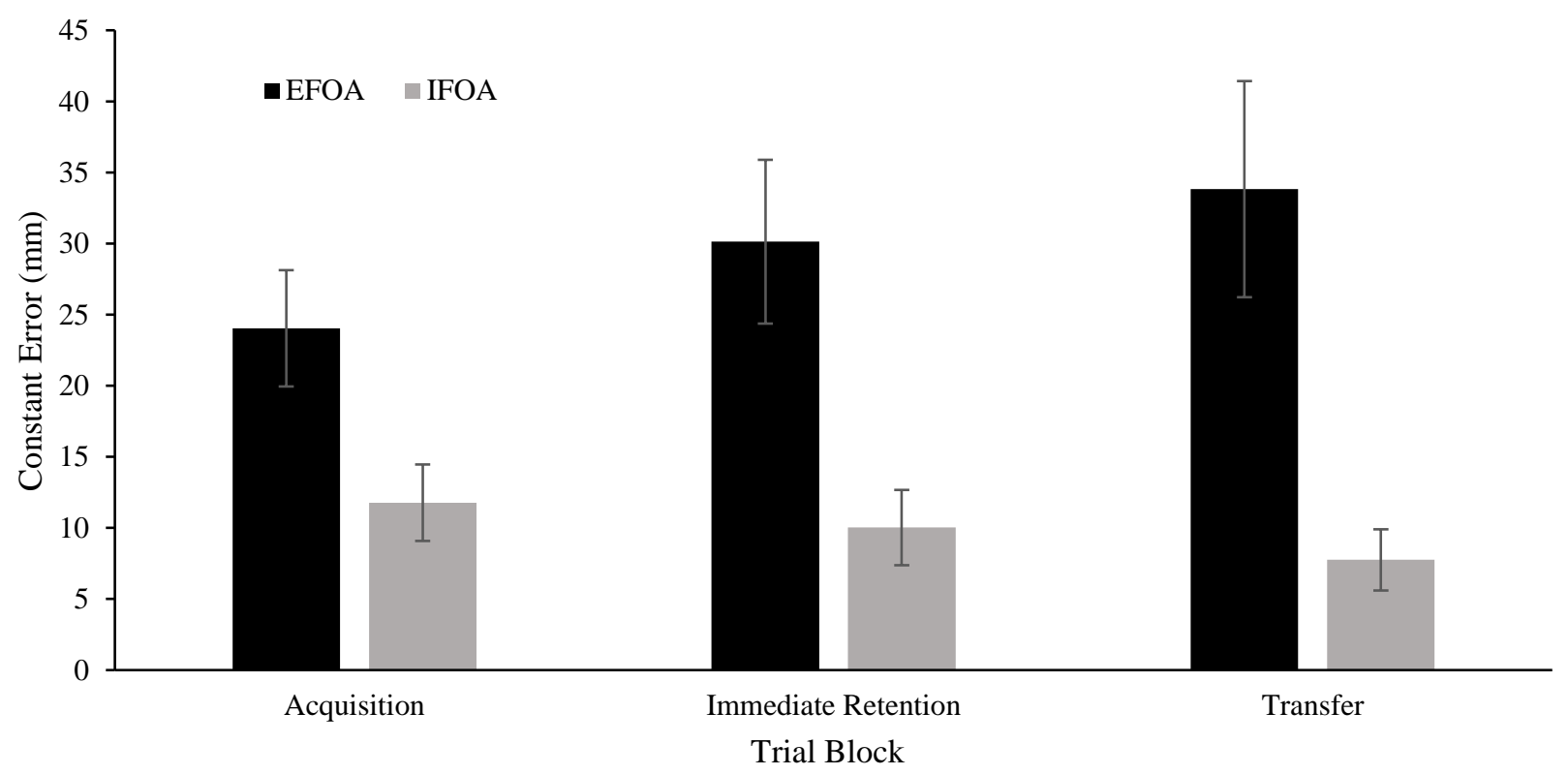

1

2 Figure 2. Experiment 1. External and internal group means (+/-1 SEm) endpoint amplitude CE at 3 acquisition, immediate retention, and transfer.

4

5

6

7

8

9

10

11

12

13

14

15

16

17 


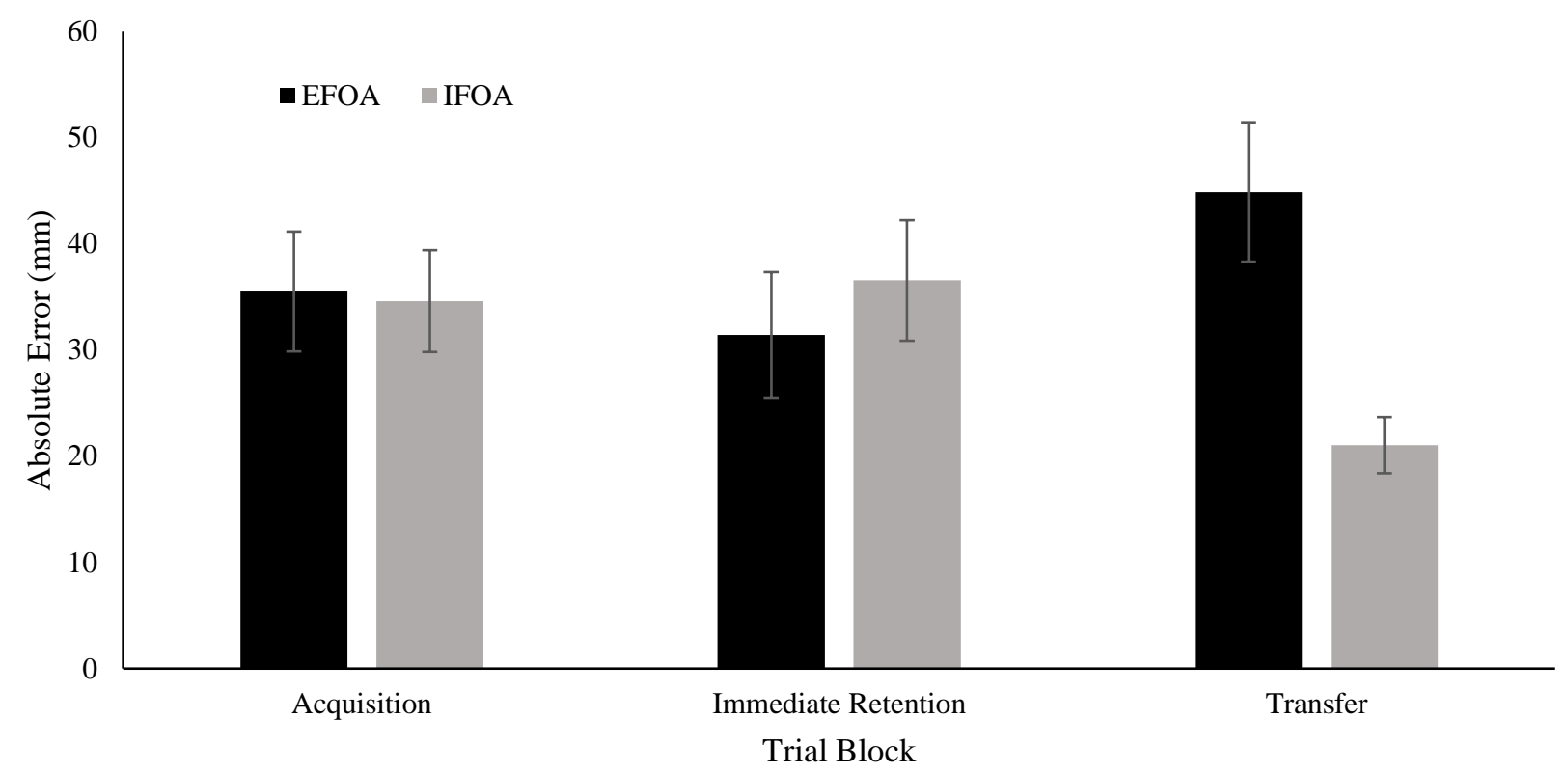

1 Figure 3. Experiment 2. External and internal group means (+/-1 SEm) endpoint amplitude AE at 3 acquisition, immediate retention, and transfer.

4

5

6

7

9

10

11 


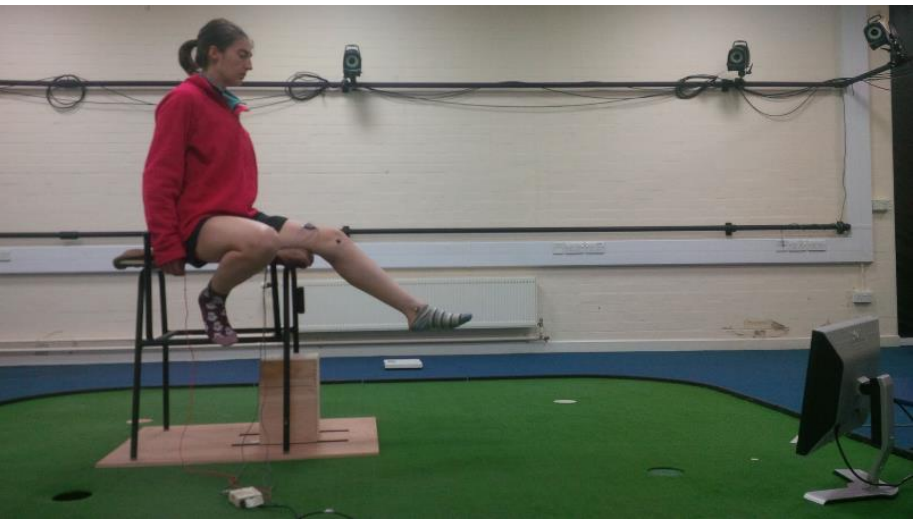

2 Figure 4. Experiment 3. This was a leg-extension task whereby participants had to extend their leg 3 from a start position to a target position. Participants were required to reproduce a pre-encoded 4 target position as accurately as possible.

5

6

7

8

9 

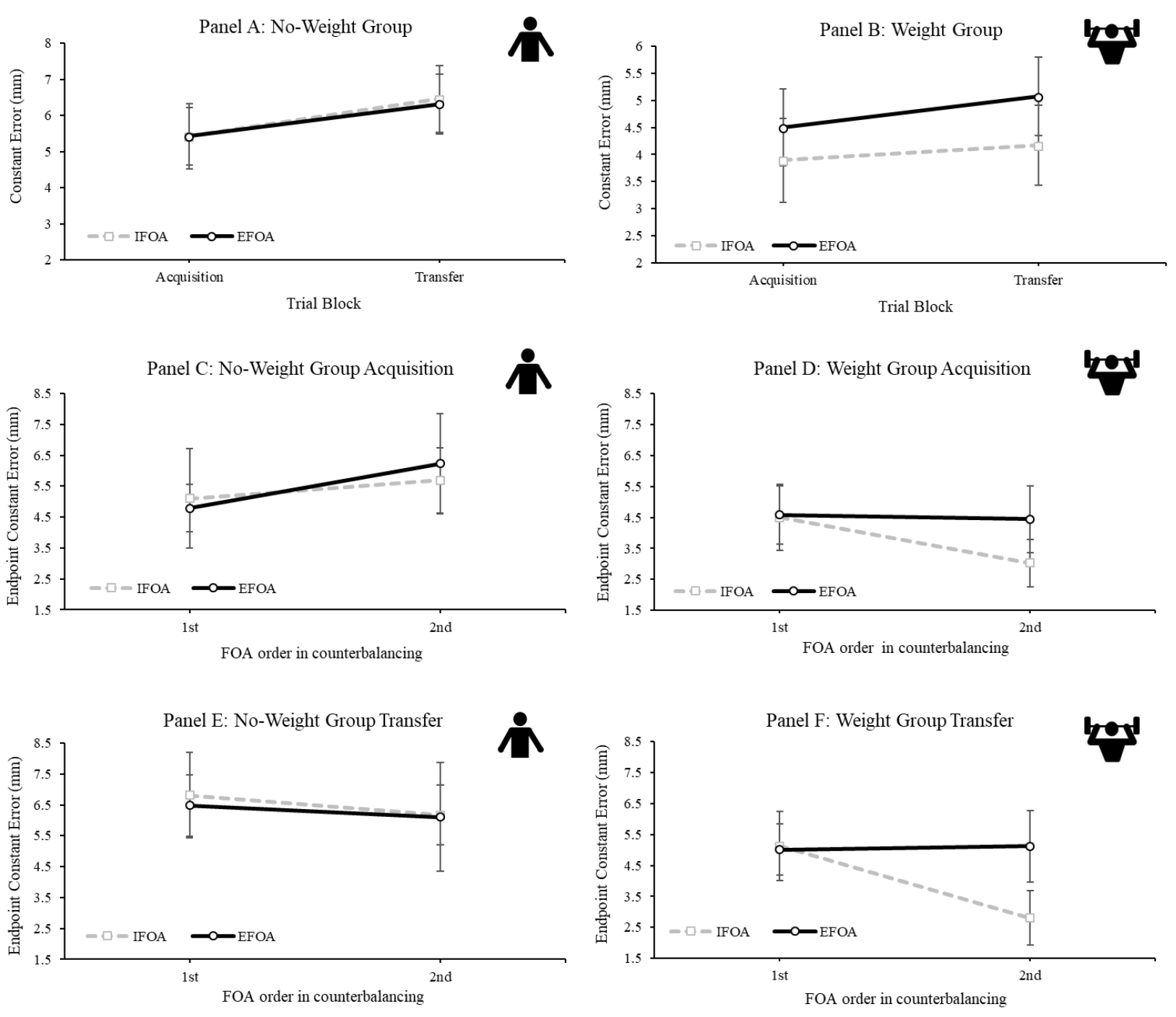

Figure 5. Experiment 3. Panel A and B show CE for the internal and external focus conditions at acquisition and transfer for the no-weight and weight groups, respectively. Panels C, D, E and F show $\mathrm{CE}$ for each focus of attention when they appear as the first (i.e., initial) focus of attention in the counterbalanced order, and when they occur second. Panels $\mathrm{C}$ and $\mathrm{E}$ display the no-weight group's acquisition and transfer, respectively. Panels D and F display the weight group's acquisition and transfer, respectively. Error bars equal +/- 1 standard error. 
1

2

4

6

7

10

11

12

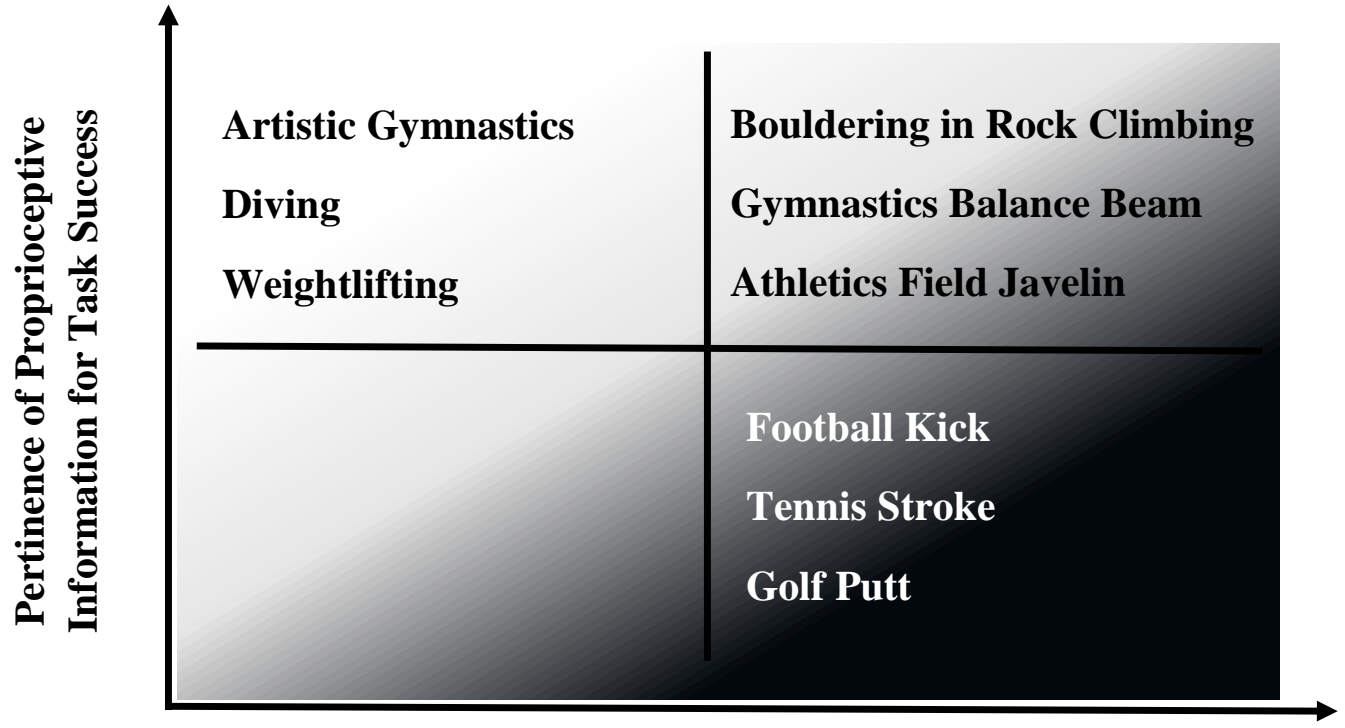

Pertinence of Visual Information for Task Success

Figure 6. The above image categorises example sports based on the pertinence of proprioceptive and visual afferent information for successful task execution. Based on the findings, the authors would advocate internal instructions and feedback for tasks high in pertinence of proprioceptive information but low in pertinence of visual information (highlighted in lighter background). For tasks low in pertinence of proprioceptive information but high in pertinence of visual information (highlighted in darker background) we would advocate external instructions and feedback. 


\section{$1 \quad$ Table 1}

2 Descriptives and statistical results of experiment 1 omnibus analysis.

3

\begin{tabular}{llllll}
\multicolumn{5}{c}{ Table 1 (A): Descriptives of outcome performance } \\
\hline Component & Focus of attention & Trial Block & CE & AE & VE \\
\hline Amplitude & External & Acquisition & $24.04(18.29)$ & $26.08(16.79)$ & $16.67(6.52)$ \\
& & Retention & $30.13(25.76)$ & $31.78(25.75)$ & $14.76(12.27)$ \\
& & Transfer & $33.83(33.99)$ & $36.09(32.62)$ & $16.19(8.85)$ \\
& Ancquisition & $11.77(12.03)$ & $14.17(10.94)$ & $11.24(4.10)$ \\
& Internal & $10.02(11.84)$ & $12.16(11.05)$ & $9.04(2.38)$ \\
& & Retention & $10.66(8.20)$ & $9.89(3.99)$ \\
& & Transfer & $.75(9.62)$ & $6.78(1.58)$ & $8.41(2.04)$ \\
Directional & Acquisition & $.512(1.32)$ & $6.76(3.10)$ & $8.04(3.73)$ \\
& External & & $-0.96(3.44)$ & $8.56(3.75)$ & $10.17(3.73)$ \\
& & Retention & $-1.14(5.09)$ & $6.34(3.03)$ & $7.98(3.39)$ \\
& Internal & Transfer & $-.23(3.44)$ & $6.06(2.46)$ & $7.76(3.09)$ \\
& Acquisition & $-.33(3.94)$ & $6.69(2.73)$ & $8.76(3.00)$ \\
\hline & Retention & & &
\end{tabular}

\begin{tabular}{|c|c|c|c|c|}
\hline Component & Effect & $\mathrm{CE}$ & $\mathrm{AE}$ & $\mathrm{VE}$ \\
\hline Amplitude & FOA Main Effect & $F_{1.38}=11.15, p=.002, \eta_{\mathrm{p}}^{2}=.23^{*} *$ & $F_{1,38}=11.72, p=.001, \eta_{\mathrm{p}}{ }^{2}=.24 * *$ & $F_{1,38}=10.04, p=.003, \eta_{p}{ }^{2}=.21^{* * *}$ \\
\hline \multirow[t]{3}{*}{ Directional } & 2 FOA $\times 3$ Trial Block Interaction & $F_{1.56,59.40}=.39, p=.631, \eta_{\mathrm{p}}{ }^{2}=.01$ & $F_{1.45,55.14}=1.78, p=.185, \eta_{\mathrm{p}}{ }^{2}=.05$ & $F_{2,76}=.54, p=.586, \eta_{\mathrm{p}}^{2}=.01$ \\
\hline & FOA Main Effect & $F_{1,38}=.45, p=.504, \eta_{\mathrm{p}}^{2}=.01$ & $F_{1,38}=.1 .69, p=.202, \eta_{p}^{2}=.04$ & $F_{1,38}=1.04, p=.314, \eta_{\mathrm{P}}{ }^{2}=.03$ \\
\hline & Trial Block Main Effect & $F_{1.56,59.40}=2.63, p=.093, \eta_{\mathrm{p}}{ }^{2}=.07$ & $F_{1.45 .55 .14}=5.38, p=.014, \eta_{p}{ }^{2}=.12^{* * *}$ & $F_{2,76}=5.95, p=.004, \eta_{\mathrm{p}}{ }^{2}=.14 * *$ \\
\hline
\end{tabular}

4

Table 1 (C): Descriptives of variability for planning and control contributions

\begin{tabular}{lllllll}
\hline Component & Focus of attention & Trial Block & VE at PkA & VE at PkV & VE at PkNA & VE at Endpoint \\
\hline Amplitude & External & Acquisition & $12.23(4.62)$ & $20.39(4.75)$ & $23.03(4.83)$ & $16.67(6.52)$ \\
& & Retention & $12.95(4.47)$ & $19.09(6.37)$ & $21.25(10.72)$ & $14.76(12.27)$ \\
& & Transfer & $15.45(4.92)$ & $22.28(5.74)$ & $22.01(6.03)$ & $16.19(8.85)$ \\
& Internal & Acquisition & $8.91(3.32)$ & $14.85(2.76)$ & $19.77(4.14)$ & $11.24(4.10)$ \\
& & Retention & $8.03(2.63)$ & $13.98(2.91)$ & $16.53(2.61)$ & $9.04(2.38)$ \\
& & Transfer & $11.83(6.53)$ & $16.14(3.77)$ & $18.20(5.26)$ & $9.89(3.99)$ \\
\hline Component & Focus of attention & Trial Block & VE at 25\% & VE at 50\% & VE at 75\% & VE at $100 \%$ \\
\hline Directional & External & Acquisition & $3.73(.78)$ & $5.42(1.26)$ & $6.93(1.60)$ & $8.41(2.04)$ \\
& & Retention & $3.53(1.23)$ & $5.15(1.87)$ & $6.51(2.66)$ & $8.04(3.73)$ \\
& & $3.98(1.05)$ & $6.11(1.74)$ & $8.27(2.73)$ & $10.17(3.73)$ \\
& Transfer & $3.56(1.02)$ & $5.24(1.66)$ & $6.86(2.33)$ & $7.98(3.39)$ \\
& Internal & Acquisition & $3.17(1.14)$ & $4.71(1.57)$ & $6.07(1.84)$ & $7.47(2.35)$ \\
& & Retention & $3.76(1.09)$ & $5.84(1.61)$ & $7.47(2.27)$ & $8.76(3.00)$ \\
\hline
\end{tabular}

Table 1 (D): Statistics of variability for planning and control contributions

\begin{tabular}{|c|c|c|c|c|c|}
\hline \multicolumn{6}{|c|}{ Offline Planning } \\
\hline Component & Effect & VE at PkA and PkV & Component & Effect & VE at $25 \%$ Movement Dist. \\
\hline \multirow[t]{7}{*}{ Amplitude } & 2 FOA $\times 3$ Trial Block $\times 2$ Marker & $F_{1.76}=.84, p=.438, \eta_{\mathrm{p}}^{2}=.02$ & Directional & 2 FOA x 3 Trial Block & $F_{2,76}=.20, p=.816, \eta_{\mathrm{p}}{ }^{2}=.05$ \\
\hline & 2 FOA $\times 3$ Trial Block & $F_{2,76}=1.93, p=.879, \eta_{p}{ }^{2}<.01$ & & FOA Main Effect & $F_{2,76}=.79, p=.380, \eta_{\mathrm{p}}{ }^{2}=.02$ \\
\hline & 2 FOA $\times 2$ Marker & $F_{1,38}=1.27, p=.267, \eta_{\mathrm{p}}{ }^{2}=.03$ & & Trial Block Main Effect & $F_{2,76}=5.30, p=.007, \eta_{\mathrm{p}}{ }^{2}=.12^{* *}$ \\
\hline & 3 Trial Block $\times 2$ Marker & $F_{1,76}=1.18, p=.313, \eta_{\mathrm{p}}^{2}=.03$ & & & \\
\hline & FOA Main Effect & $F_{1,38}=30.62, p<.001, \eta_{\mathrm{p}}^{2}=.45^{* *}$ & & & \\
\hline & Trial Block Main Effect & $F_{2,76}=12.66, p<.001, \eta_{\mathrm{P}}{ }^{2}=.25^{* * *}$ & & & \\
\hline & Marker Main Effect & $F_{1,38}=72.35, p<.001, \eta_{\mathrm{P}}^{2}=.66^{* *}$ & & & \\
\hline \multicolumn{6}{|c|}{ Online Control } \\
\hline Component & Effect & VE at PkV, PkNA, and endpoint & Component & Effect & VE at 75 and $100 \%$ Movment Dist. \\
\hline \multirow[t]{7}{*}{ Amplitude } & 2 FOA $\times 3$ Trial Block $x 3$ Markers & $F_{3.24,123.05}=.22, p=.895, \eta_{\mathrm{p}}{ }^{2}=.01$ & Directional & 2 FOA $\times 3$ Trial Block $\times 2$ Distance & $F_{2,76}=.56, p=.58, \eta_{\mathrm{p}}{ }^{2}=.01$ \\
\hline & 2 FOA $\times 3$ Trial Block & $F_{2,76}=.14, p=.870, \eta_{\mathrm{P}}^{2}<.01$ & & 2 FOA $\times 3$ Trial Block & $F_{2,76}=.507, p=.604, \eta_{\mathrm{p}}{ }^{2}=.01$ \\
\hline & 2 FOA x 2 Marker & $F_{2,76}=.80, p=.455, \eta_{\mathrm{P}}{ }^{2}=.02$ & & 2 FOA $\times 2$ Distance & $F_{1,38}=1.33, p=.256, \eta_{\mathrm{p}}{ }^{2}=.03$ \\
\hline & 3 Trial Block x 2 Marker & $F_{3.24,123.05}=1.67, p=.173, \eta_{\mathrm{p}}{ }^{2}=.04$ & & 3 Trial Block x 2 Distance & $F_{2,76}=.84, p=.437, \eta_{\mathrm{p}}^{2}=.02$ \\
\hline & FOA Main Effect & $F_{138}=16.80, p<.001, \eta_{\mathrm{p}}{ }^{2}=.31 * *$ & & FOA Main Effect & $F_{1,38}=.86, p=.359, \eta_{\mathrm{p}}^{2}=.02$ \\
\hline & Trial Block Main Effect & $F_{2,76}=5.02, p=.001, \eta_{\mathrm{p}}{ }^{2}=.12^{* *}$ & & Trial Block Main Effect & $F_{2,76}=7.36, p=.001, \eta_{\mathrm{p}}{ }^{2}=.16^{* *}$ \\
\hline & Marker Main Effect & $F_{2,76}=39.93, p<.001, \eta_{\mathbb{P}}^{2}=.51^{* *}$ & & Distance Main Effect & $F_{1,38}=86.66, p<.001, \eta_{\mathrm{p}}{ }^{2}=.70^{* *}$ \\
\hline
\end{tabular}

7

$8 * \mathrm{p}<.052 . * * \mathrm{p}<.05$.

9 


\section{$1 \quad$ Table 2}

2 Descriptives and statistical results of experiment 2 omnibus analysis.

\begin{tabular}{|c|c|c|c|c|c|}
\hline Component & Focus of attention & Trial Block & $\mathrm{CE}$ & $\mathrm{AE}$ & $\mathrm{VE}$ \\
\hline \multirow[t]{6}{*}{ Amplitude } & \multirow[t]{3}{*}{ External } & Acquisition & $-7.30(40.71)$ & $35.49(25.24)$ & $23.49(7.44)$ \\
\hline & & Retention & $24.03(29.36)$ & 31.41 (26.42) & $17.76(8.48)$ \\
\hline & & Transfer & $-35.17(38.68)$ & 44.86 (29.33) & $26.90(25.77)$ \\
\hline & \multirow[t]{3}{*}{ Internal } & Acquisition & $-3.07(40.11)$ & 34.59 (21.45) & $24.12(7.52)$ \\
\hline & & Retention & 30.71 (29.77) & $36.53(25.38)$ & $18.36(9.70)$ \\
\hline & & Transfer & $9.12(18.50)$ & $21.02(11.83)$ & $9.78(5.43)$ \\
\hline \multirow[t]{6}{*}{ Directional } & \multirow[t]{3}{*}{ External } & Acquisition & $.92(10.91)$ & 36.35 (22.28) & $42.81(28.09)$ \\
\hline & & Retention & $-3.31(12.79)$ & $31.47(24.02)$ & $35.15(30.20)$ \\
\hline & & Transfer & $2.91(14.63)$ & 34.66 (23.96) & $39.37(30.21)$ \\
\hline & \multirow[t]{3}{*}{ Internal } & Acquisition & $-3.03(5.94)$ & $22.32(10.13)$ & $27.13(12.26)$ \\
\hline & & Retention & $-5.42(10.96)$ & $19.33(12.03)$ & $22.04(13.63)$ \\
\hline & & Transfer & $-2.45(12.55)$ & $22 . .06(15.71)$ & 25.21 (17.37) \\
\hline
\end{tabular}

\begin{tabular}{|c|c|c|c|c|}
\hline Component & Effect & $\mathrm{CE}$ & $\mathrm{AE}$ & VE \\
\hline \multirow[t]{3}{*}{ Amplitude } & 2 FOA $\times 3$ Trial Block Interaction & $F_{1.36,51.82}=1.489, p=.232, \eta_{\mathrm{P}}^{2}=.04$ & $F_{1.37,51,90}=8.54, p=.002, \eta_{\mathrm{p}}{ }^{2}=.18^{* * *}$ & $F_{1.49,56.51}=2.54, p=.103, \eta_{\mathrm{p}}{ }^{2}=.06$ \\
\hline & FOA Main Effect & $F_{1,38}=14.29, p=.001, \eta_{\mathrm{p}}^{2}=.27 * *$ & $F_{1,38}=1.09, p=.302, \eta_{\mathrm{p}}^{2}=.03$ & $F_{1,38}=.29, p=.592, \eta_{\mathrm{p}}^{2}=.008$ \\
\hline & Trial Block Main Effect & $F_{1.36,51.82}=25.301, p<.001, \eta_{\mathrm{p}}{ }^{2}=.40^{* *}$ & $F_{1.37,51.90}=.16, p=.767, \eta_{\mathrm{P}}{ }^{2}=.004$ & $F_{1.49,56.51}=5.08, p=.016, \eta_{\mathrm{p}}{ }^{2}=.12 * *$ \\
\hline \multirow[t]{3}{*}{ Directional } & 2 FOA $\times 3$ Trial Block Interaction & $F_{1.59,60282}=.16, p=.851, \eta_{\mathrm{p}}{ }^{2}=.004$ & $F_{1.63 .61 .90}=.25, p=.732, \eta_{\mathrm{p}}{ }^{2}=.007$ & $F_{1.616,61.20}=.346, p=.708, \eta_{\mathrm{p}}{ }^{2}=.009$ \\
\hline & FOA Main Effect & $F_{1.38}=2.07, p=.159, \eta_{\mathrm{p}}^{2}=.05$ & $F_{1,38}=5.04, p=.031, \eta_{\mathrm{p}}^{2}=.12^{* *}$ & $F_{1,38}=4.02, p=.052, \eta_{\mathrm{p}}^{2}=.10^{*}$ \\
\hline & Trial Block Main Effect & $F_{1.59,6028}=2.77, p=.082, \eta_{p}{ }^{2}=.07$ & $F_{1.63,6190,}=4.37, p=.023, \eta_{\mathrm{p}}{ }^{2}=.10^{* * *}$ & $F_{1.61,61.20}=8.46, p=.001, \eta_{\mathrm{p}}^{2}=.18^{* *}$ \\
\hline
\end{tabular}

\section{4}

Table 2 (C): Descriptives of variability for planning and control contributions

\begin{tabular}{lllllll}
\multicolumn{7}{c}{ Table 2 (C): Descriptives of variability for planning and control contributions } \\
\hline Component & Focus of attention & Trial Block & VE at PkA & VE at PkV & VE at PkNA & VE at Endpoint \\
\hline Amplitude & External & Acquisition & $12.50(8.08)$ & $17.11(3.36)$ & $25.09(4.23)$ & $23.49(7.44)$ \\
& & Retention & $11.08(6.44)$ & $15.27(5.28)$ & $21.00(9.31)$ & $17.76(8.48)$ \\
& & Transfer & $15.00(14.03)$ & $18.07(11.08)$ & $28.23(23.22)$ & $26.90(25.80)$ \\
& Internal & Acquisition & $14.25(5.76)$ & $20.21(4.28)$ & $27.56(8.27)$ & $24.76(7.52)$ \\
& & Retention & $11.77(4.29)$ & $17.12(4.80)$ & $22.93(9.25)$ & $18.36(9.70)$ \\
& & Transfer & $13.06(4.88)$ & $18.73(4.88)$ & $23.29(6.50)$ & $19.78(5.43)$ \\
\hline Component & Focus of attention & Trial Block & VE at 25\% & VE at 50\% & VE at 75\% & VE at 100\% \\
\hline Directional & External & Acquisition & $11.67(6.74)$ & $22.84(14.59)$ & $34.36(23.02)$ & $42.81(28.09)$ \\
& & Retention & $10.02(8.00)$ & $19.20(17.23)$ & $28.94(26.83)$ & $35.15(30.20)$ \\
& & Transfer & $12.09(9.40)$ & $23.89(21.07)$ & $34.58(29.13)$ & $39.37(30.21)$ \\
& \multirow{3}{*}{ Internal } & Acquisition & $7.06(2.99)$ & $12.89(5.97)$ & $19.87(9.53)$ & $27.13(12.26)$ \\
& & Retention & $5.61(2.84)$ & $10.50(6.21)$ & $16.06(10.20)$ & $22.04(13.63)$ \\
& & Transfer & $6.37(3.50)$ & $11.66(7.43)$ & $17.989(12.71)$ & $25.21(17.37)$ \\
\hline
\end{tabular}

Table 2 (D): Statistics of variability for planning and control contribution

\begin{tabular}{|c|c|c|c|c|c|}
\hline \multicolumn{6}{|c|}{ Offline Planning } \\
\hline Component & Effect & VE at PkA and PkV & Component & Effect & VE at $25 \%$ Movement Dist. \\
\hline \multirow[t]{7}{*}{ Amplitude } & 2 FOA $\times 3$ Trial Block $\times 2$ Marker & $F_{2,76}=.22, p=.802, \eta_{\mathrm{p}}{ }^{2}=.01$ & Directional & 2 FOA $\times 3$ Trial Block & $F_{1.49,56.55}=1.18, p=.303, \eta_{\mathrm{p}}{ }^{2}=.03$ \\
\hline & 2 FOA $\times 3$ Trial Block & $F_{1.44,33.32}=2.00, p=.157, \eta_{\mathrm{p}}{ }^{2}=.05$ & & FOA Main Effect & $F_{1,38}=6.91, p=.012, \eta_{\mathrm{p}}{ }^{2}=.15^{* *}$ \\
\hline & 2 FOA x 2 Marker & $F_{1,38}=.79, p=.380, \eta_{\mathrm{p}}{ }^{2}=.02$ & & Trial Block Main Effect & $F_{1.49,56.55}=6.94, p=.005, \eta_{\mathrm{p}}{ }^{2}=.15^{* *}$ \\
\hline & 3 Trial Block $x 2$ Marker & $F_{2,76}=.30, p=.740, \eta_{\mathrm{p}}{ }^{2}=.01$ & & & \\
\hline & FOA Main Effect & $F_{1,38}=.36, p=.552, \eta_{\mathrm{p}}{ }^{2}=.01$ & & & \\
\hline & Trial Block Main Effect & $F_{1.44,3332}=5.95, p=.010, \eta_{p}{ }^{2}=.14 * *$ & & & \\
\hline & Marker Main Effect & $F_{1,38}=25.18, p<.001, \eta_{p}^{2}=.40^{* *}$ & & & \\
\hline \multicolumn{6}{|c|}{ Online Control } \\
\hline 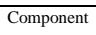 & Effect & VE at PkV, PkNA, and endpoint & Component & Effect & VE at 75 and $100 \%$ Movment Dist. \\
\hline \multirow[t]{7}{*}{ Amplitude } & 2 FOA $\times 3$ Trial Block $\times 3$ Markers & $F_{231,87.81}=1.82, p=.162, \eta_{\mathrm{p}}{ }^{2}=.05$ & Directional & 2 FOA $\times 3$ Trial Block $\times 2$ Distance & $F_{1.31 .52 .12}=4.35, p=.032, \eta_{\mathrm{p}}{ }^{2}=.10$ \\
\hline & 2 FOA $\times 3$ Trial Block & $F_{1.32,50.15}=2.22, p=.136, \eta_{\mathrm{p}}{ }^{2}=.06$ & & 2 FOA $\times 3$ Trial Block & $F_{1.59,60.21}=.39, p=.630, \eta_{\mathrm{P}}{ }^{2}=.01$ \\
\hline & 2 FOA x 2 Marker & $F_{1.46,50.54}=1.71, p=.196, \eta_{p}{ }^{2}=.04$ & & 2 FOA $\times 2$ Distance & $F_{1,38}=.06, p=.810, \eta_{\mathrm{P}}{ }^{2}<.01$ \\
\hline & 3 Trial Block x 2 Marker & $F_{2.31,87.81}=1.80, p=.165, \eta_{p}{ }^{2}=.05$ & & 3 Trial Block $\mathrm{x} 2$ Distance & $F_{1.31,49.71}=5.34, p=.017, \eta_{p}{ }^{2}=.12^{* *}$ \\
\hline & FOA Main Effect & $F_{1,38}<.01, p=.993, \eta_{\mathrm{p}}{ }^{2}<.01$ & & FOA Main Effect & $F_{11,38}=4.77, p=.035, \eta_{p}^{2}=.11^{* *}$ \\
\hline & Trial Block Main Effect & $F_{1.32,50.15}=4.47, p=.030, \eta_{\mathrm{p}}{ }^{2}=.11^{* *}$ & & Trial Block Main Effect & $F_{1.59,6021}=7.28, p=.003, \eta_{\mathrm{P}}{ }^{2}=.16^{* *}$ \\
\hline & Marker Main Effect & $F_{1.46 .50 .54}=25.23, p<.001, \eta_{p}{ }^{2}=.40^{* *}$ & & Distance Main Effect & $F_{1.38}=92.82, p<.001, \eta_{p}{ }^{2}=.71 * *$ \\
\hline
\end{tabular}

$* \mathrm{p}<.052 . * * \mathrm{p}<.05$ 


\section{$1 \quad$ Table 3}

\section{Descriptives and statistical results of experiment 3 omnibus analysis.}

Table 3 (A): Descriptives of outcome performance

\begin{tabular}{|c|c|c|c|c|c|c|}
\hline Ankle & Focus of attention & Order & Trial Block & $\mathrm{CE}$ & $\mathrm{AE}$ & $\mathrm{VE}$ \\
\hline \multirow[t]{8}{*}{ Weighted } & External & before an internal FOA & Acquisition & $4.58(2.47)$ & $5.13(2.28)$ & $3.75(1.29)$ \\
\hline & & & Transfer & $5.01(2.19)$ & $5.26(2.02)$ & $3.57(.67)$ \\
\hline & & after an internal FOA & Acquisition & $4.44(3.42)$ & $5.30(2.64)$ & $3.53(.70)$ \\
\hline & & & Transfer & $5.12(2.19)$ & $5.79(3.01)$ & $3.81(1.19)$ \\
\hline & Internal & before an external FOA & Acquisition & $4.50(3.39)$ & $5.34(2.58)$ & $3.39(1.09)$ \\
\hline & & & Transfer & $5.12(3.52)$ & $5.86(2.81)$ & $4.05(1.01)$ \\
\hline & & after an external FOA & Acquisition & $3.02(2.04)$ & $4.19(1.18)$ & $3.76(.71)$ \\
\hline & & & Transfer & $2.80(2.31)$ & $3.80(1.58)$ & $3.49(.95)$ \\
\hline \multirow[t]{4}{*}{ No-Weight } & External & before an internal FOA & Acquisition & $4.79(2.41)$ & $5.19(1.99)$ & $3.45(.82)$ \\
\hline & & & Transfer & $6.49(3.14)$ & $6.82(2.86)$ & $4.17(1.11)$ \\
\hline & & after an internal FOA & Acquisition & $6.23(4.60)$ & $6.74(4.02)$ & $3.81(.94)$ \\
\hline & & & Transfer & $6.11(4.97)$ & $6.58(4.57)$ & $3.93(1.30)$ \\
\hline
\end{tabular}

Table 3 (B): Statistics of outcome performance

\begin{tabular}{|c|c|c|c|c|}
\hline Ankle & Effect & $\mathrm{CE}$ & $\mathrm{AE}$ & $\mathrm{VE}$ \\
\hline \multirow[t]{7}{*}{ Weighted } & 2 FOA $\times 2$ Block $\times 2$ Order Interaction & $F_{1,15}=.34, p=.567, \eta_{\mathrm{P}}^{2}=.02$ & $F_{1,15}=.33, p=.575, \eta_{\mathrm{p}}^{2}=.02$ & $F_{1,15}=.59, p=.456, \eta_{\mathrm{p}}^{2}=.04$ \\
\hline & 2 FOA $\times 2$ Trial Block Interaction & $F_{1,15}=.48, p=.501, \eta_{\mathrm{p}}^{2}=.03$ & $F_{1,15}=.26, p=.616, \eta_{\mathrm{p}}^{2}=.02$ & $F_{1,15}=.24, p=.631, \eta_{\mathrm{p}}^{2}=.02$ \\
\hline & 2 FOA $\times 2$ Order Interaction & $F_{1,15}=4.74, p=.046, \eta_{\mathrm{p}}{ }^{2}=.24 * *$ & $F_{1,15}=3.17, p=.095, \eta_{\mathrm{P}}^{2}=.18$ & $F_{1,15}=.05, p=.821, \eta_{\mathrm{p}}{ }^{2}<.01$ \\
\hline & 2 Block $\times 2$ Order Interaction & $F_{1,15}=.44, p=.517, \eta_{\mathrm{P}}^{2}=.029$ & $F_{1,15}=.81, p=.382, \eta_{\mathrm{p}}^{2}=.05$ & $F_{1,15}=2.86, p=.112, \eta_{\mathrm{p}}{ }^{2}=.16$ \\
\hline & FOA Main Effect & $F_{1,15}=4.45, p=.052, \eta_{\mathrm{p}}{ }^{2}=.23^{*}$ & $F_{1,15}=2.64, p=.125, \eta_{\mathrm{P}}^{2}=.15$ & $F_{1,15}<.01, p=.968, \eta_{p}{ }^{2}<.01$ \\
\hline & Trial Block Main Effect & $F_{1,15}=.83, p=.376, \eta_{\mathrm{p}}{ }^{2}=.05$ & $F_{1.15}=.29, p=.598, \eta_{\mathrm{p}}{ }^{2}=.02$ & $F_{1,15}=.38, p=.550, \eta_{\mathrm{P}}{ }^{2}=.02$ \\
\hline & Order Main Effect & $F_{1,15}=.48, p=.498, \eta_{\mathrm{P}}{ }^{2}=.03$ & $F_{1,15}=.84, p=.374, \eta_{\mathrm{p}}{ }^{2}=.05$ & $F_{1,15}=.02, p=.891, \eta_{p}{ }^{2}<.01$ \\
\hline \multirow[t]{7}{*}{ No-Weight } & 2 FOA $\times 2$ Block $\times 2$ Order Interaction & $F_{1,16}=4.47, p=.051, \eta_{\mathrm{p}}{ }^{2}=.22^{*}$ & $F_{1,16}=3.66, p=.074, \eta_{\mathrm{p}}^{2}=.17$ & $F_{1,16}=.01, p=.912, \eta_{\mathrm{p}}^{2}<.01$ \\
\hline & 2 FOA $\times 2$ Trial Block Interaction & $F_{1,16}=.19, p=.671, \eta_{\mathrm{P}}{ }^{2}=.01$ & $F_{1,16}=.13, p=.723, \eta_{\mathrm{p}}^{2}=.01$ & $F_{1,16}=.06, p=.808, \eta_{\mathrm{p}}^{2}<.01$ \\
\hline & 2 FOA $\times 2$ Order Interaction & $F_{1,16}=.20, p=.660, \eta_{\mathrm{P}}^{2}=.01$ & $F_{1,16}=.07, p=.793, \eta_{\mathrm{p}}<.01$ & $F_{1,16}=.18, p=.675, \eta_{\mathrm{P}}{ }^{2}=.01$ \\
\hline & 2 Block $\times 2$ Order Interaction & $F_{1,16}=.01, p=.943, \eta_{\mathrm{P}}^{2}<.01$ & $F_{1,16}=.49, p=.494, \eta_{\mathrm{P}}^{2}=.03$ & $F_{1,16}=4.02, p=.062, \eta_{\mathrm{P}}^{2}=.21$ \\
\hline & FOA Main Effect & $F_{1,16}=.15, p=.708, \eta_{\mathrm{p}}{ }^{2}=.01$ & $F_{1,16}=.06, p=.815, \eta_{\mathrm{p}}^{2}<.01$ & $F_{1,16}<.01, p=.991, \eta_{\mathrm{p}}^{2}<.01$ \\
\hline & Trial Block Main Effect & $F_{1,16}=5.73, p=.029, \eta_{\mathrm{p}}^{2}=.26^{* *}$ & $F_{1,16}=5.81, p=.028, \eta_{\mathrm{p}}^{2}=.27^{* * *}$ & $F_{1,16}=8.36, p=.011, \eta_{\mathrm{p}}{ }^{2}=.34^{* *}$ \\
\hline & Order Main Effect & $F_{1,16}=.03, p=.864, \eta_{\mathrm{p}}^{2}<.01$ & $F_{1,16}=.137, p=.716, \eta_{p}^{2}=.01$ & $F_{1.16}<.01, p=.971, \eta_{\mathrm{p}}^{2}<.01$ \\
\hline
\end{tabular}

Table 3 (C): Descriptives of variability for planning and control contributions

\begin{tabular}{|c|c|c|c|c|c|c|c|}
\hline Ankle & Focus of attention & Order & Trial Block & VE at PkA & $\mathrm{VE}$ at $\mathrm{PkV}$ & VE at PkNA & VE at Endpoint \\
\hline \multirow[t]{8}{*}{ Weighted } & External & before an internal FOA & Acquisition & $1.62(.50)$ & $4.37(.91)$ & $7.26(1.15)$ & $7.49(1.01)$ \\
\hline & & & Transfer & $1.76(.67)$ & $5.56(.69)$ & $9.99(1.05)$ & $11.28(1.10)$ \\
\hline & & after an internal FOA & Acquisition & $1.98(.88)$ & $4.16(.68)$ & $7.09(.91)$ & $7.64(1.06)$ \\
\hline & & & Transfer & $2.12(.73)$ & $6.03(.83)$ & $10.41(1.39)$ & $11.51(1.62)$ \\
\hline & Internal & before an external FOA & Acquisition & $2.19(1.09)$ & $4.63(.65)$ & $7.54(1.01)$ & $8.19(.84)$ \\
\hline & & & Transfer & $2.50(.80)$ & $5.84(.93)$ & $10.03(.98)$ & $11.12(1.28)$ \\
\hline & & after an external FOA & Acquisition & $1.57(.52)$ & $4.26(.80)$ & $7.21(1.36)$ & $7.74(1.15)$ \\
\hline & & & Transfer & $1.45(.51)$ & $5.71(.63)$ & $9.98(.97)$ & $11.20(1.22)$ \\
\hline \multirow[t]{8}{*}{ No-Weight } & External & before an internal FOA & Acquisition & $2.04(.71)$ & $4.57(.71)$ & $7.45(.97)$ & $7.52(1.06)$ \\
\hline & & & Transfer & $2.28(.57)$ & $5.28(.76)$ & $9.21(.63)$ & $10.64(1.23)$ \\
\hline & & after an internal FOA & Acquisition & $1.72(.90)$ & $4.50(.96)$ & $7.40(1.00)$ & $8.15(1.41)$ \\
\hline & & & Transfer & $1.88(.63)$ & $6.03(.97)$ & $10.78(1.25)$ & $12.05(2.26)$ \\
\hline & Internal & before an external FOA & Acquisition & $2.48(1.81)$ & $5.25(1.34)$ & $8.37(1.45)$ & $8.84(1.51)$ \\
\hline & & & Transfer & $2.36(.79)$ & $5.97(1.14)$ & $10.32(1.30)$ & $11.49(1.73)$ \\
\hline & & after an external FOA & Acquisition & $1.78(.72)$ & $3.88(.91)$ & $6.90(.68)$ & $7.41(1.17)$ \\
\hline & & & Transfer & $1.71(.79)$ & $5.39(.70)$ & $9.41(.91)$ & $10.63(1.38)$ \\
\hline
\end{tabular}

\begin{tabular}{|c|c|c|c|c|c|}
\hline \multicolumn{3}{|c|}{$\begin{array}{l}\text { Offline Planning } \\
\end{array}$} & \multicolumn{3}{|c|}{ Online Control } \\
\hline Ankle & Effect & VE at PkA and PkV & Ankle & Effect & VE at $P k V, P k N A$, and endpoint \\
\hline \multirow[t]{8}{*}{ Weighted } & 2 FOA $x 2$ Trial Block $x 2$ Marker $x 2$ Order & $F_{2,15}=16.22, p=.001, \eta_{p}{ }^{2}=.52 * *$ & Weighted & 2 FOA $\times 2$ Trial Block $x 3$ Marker $x 2$ Order & $F_{2,30}=.16, p=.851, \eta_{\mathrm{P}}{ }^{2}=.01$ \\
\hline & 2 FOA x 2 Trial Block x 2 Marker & $F_{2,15}=.78, p=.391, \eta_{\mathbb{P}}{ }^{2}=.05$ & & 2 FOA x 2 Trial Block x 3 Marker & $F_{2,30}=1.18, p=.322, \eta_{\mathrm{p}}{ }^{2}=.07$ \\
\hline & 2 FOA x 2 Marker x 2 Order & $F_{2,15}=1.06, p=.319, \eta_{\mathrm{P}}{ }^{2}=.07$ & & 2 FOA $\times 3$ Marker $\times 2$ Order & $F_{2,30}=.06, p=.941, \eta_{\mathrm{p}}{ }^{2}<.01$ \\
\hline & 2 FOA x 2 Marker & $F_{2,15}=.02, p=.896, \eta_{p}{ }^{2}<.01$ & & 2 FOA x 3 Marker & $F_{2,30}=.11, p=.895, \eta_{\mathrm{P}}{ }^{2}=.01$ \\
\hline & 2 FOA $\times 2$ Trial Block $\times 2$ Order & $F_{2,15}=.39, p=.542, \eta_{\mathbb{P}}{ }^{2}=.03$ & & 2 FOA $\times 2$ Trial Block $\times 2$ Order & $F_{1,15}=2.12, p=.166, \eta_{\mathrm{p}}{ }^{2}=.12$ \\
\hline & 2 FOA x 2 Trial Block & $F_{2,15}=.37, p=.552, \eta_{\mathbb{p}}{ }^{2}=.02$ & & 2 FOA $\times 2$ Trial Block & $F_{1,15}=2.22, p=.157, \eta_{\mathrm{p}}{ }^{2}=.13$ \\
\hline & 2 FOA $\times 2$ Order & $F_{2,15}=2.17, p=.162, \eta_{\mathrm{P}}{ }^{2}=.13$ & & 2 FOA $\times 2$ Order & $F_{1,15}=.02, p=.887, \eta_{\mathrm{p}}{ }^{2}<.01$ \\
\hline & FOA Main Effect & $F_{2,15}=.44, p=.517, \eta_{\mathrm{p}}{ }^{2}=.03$ & & FOA Main Effect & $F_{1.15}=.07, p=.795, \eta_{\mathrm{P}}{ }^{2}=.01$ \\
\hline
\end{tabular}


Trial Block Main Effect

Marker Main Effect

Order Main Effect

No-Weight 2 FOA $\times 2$ Trial Block $\times 2$ Marker $\times 2$ Order

2 FOA $x 2$ Trial Block $x 2$ Marker

2 FOA $x 2$ Marker $x 2$ Order

2 FOA $x 2$ Marker

2 FOA $\times 2$ Trial Block $\times 2$ Order

2 FOA $\times 2$ Trial Block

2 FOA $\times 2$ Order

FOA Main Effect

Trial Block Main Effect

Marker Main Effect

Order Main Effect
$F_{2,15}=66.17, p<.001, \eta_{\mathrm{P}}{ }^{2}=.82 * *$

$F_{2,15}=248.90, p<.001, \eta_{\mathrm{p}}{ }^{2}=.94 * *$

$F_{2,15}=2.70, p=.121, \eta_{\mathrm{P}}^{2}=.15$

$F_{2,16}=2.15, p=.162, \eta_{\mathrm{p}}{ }^{2}=.12$

$F_{2,16}=.26, p=.618, \eta_{\mathrm{p}}{ }^{2}=.02$

$F_{2,16}=1.08, p=.315, \eta_{\mathrm{p}}{ }^{2}=.06$

$F_{2,16}=.16, p=.691, \eta_{\mathrm{p}}{ }^{2}=.01$

$F_{2,16}=4.99, p=.040, \eta_{p}{ }^{2}=.24^{* *}$

$F_{2,16}=.70, p=.415, \eta_{\mathrm{P}}^{2}=.04$

$F_{2,16}=7.40, p=.015, \eta_{\mathrm{p}}{ }^{2}=.32^{* *}$

$F_{2,16}=.17, p=.682, \eta_{\mathrm{P}}^{2}=.01$

$F_{2,16}=14.87, p=.001, \eta_{p}{ }^{2}=.48 * *$

$F_{2,16}=508.04, p<.001, \eta_{p}{ }^{2}=.97 * *$
Trial Block Main Effect

Marker Main Effect

Order Main Effect

No-Weight

2 FOA x 2 Trial Block $\times 3$ Marker x 2 Order

2 FOA $\times 2$ Trial Block $\times 3$ Marker

2 FOA $\times 3$ Marker $x 2$ Order

2 FOA $\times 3$ Marker

2 FOA $\times 2$ Trial Block $\times 2$ Order

2 FOA $\times 2$ Trial Block

2 FOA $\times 2$ Order

FOA Main Effect

Trial Block Main Effect

Marker Main Effect
$F_{1,15}=331.93, p<.001, \eta_{\mathrm{p}}{ }^{2}=.95^{* *}$

$F_{1.36,20.33}=432.73, p<.001, \eta_{\mathrm{p}}{ }^{2}=.97 * *$

$F_{2,30}=.28, p=.607, \eta_{\mathrm{P}}{ }^{2}=.02$

$F_{2,32}=.80, p=.457, \eta_{\mathrm{p}^{2}}=.05$

$F_{2,32}=1.46, p=.248, \eta_{\mathrm{p}}{ }^{2}=.08$

$F_{2,32}=1.02, p=.374, \eta_{\mathrm{p}}{ }^{2}=.06$

$F_{2,32}=.02, p=.984, \eta_{\mathrm{p}}<.01$

$F_{1,16}=10.62, p=.005, \eta_{\mathrm{p}}{ }^{2}=.40$ **

$F_{1,16}=1.37, p=.258, \eta_{\mathrm{p}}{ }^{2}=.08$

$F_{1,16}=2.58, p=.128, \eta_{\mathrm{p}}{ }^{2}=.14$

$F_{1,16}=.04, p=.853, \eta_{\mathrm{p}}^{2}<.01$

$F_{1,16}=117.18, p<.001, \eta_{\mathrm{p}}{ }^{2}=.92^{* *}$

$F_{1.25,20.02}=180.47, p<.001, \eta \eta_{\mathrm{p}}{ }^{2}=.92 * *$

1

$2 * \mathrm{p}<.052 . * * \mathrm{p}<.05$.

3

4

5 